\title{
Functional Equation for Dynamical Zeta Functions of Milnor-Thurston Type
}

\section{David Ruelle}

I.H.E.S., 35, route de Chartres, F-91440 Bures-sur-Yvette, France

Received: 8 April 1994

\begin{abstract}
A Milnor-Thurston type dynamical zeta function $\zeta_{L}(Z)$ is associated with a family of maps of the interval $(-1,1)$. Changing the direction of time produces a new zeta function $\zeta_{L}^{\prime}(Z)$. These zeta functions satisfy a functional equation $\zeta_{L}(Z) \zeta_{L}^{\prime}(\varepsilon Z)=\zeta_{0}(Z)$ (where $\varepsilon$ amounts to sign changes and, generically, $\zeta_{0} \equiv 1$ ). The functional equation has non-trivial implications for the analytic properties of $\zeta_{L}(Z)$
\end{abstract}

\section{Introduction}

Milnor and Thurston [2] have shown how the zeta function $\zeta(z)$ counting the periodic points of a piecewise monotone interval map $f$ could be expressed in terms of a kneading determinant $D(z)$. The zeta function considered by Milnor and Thurston is closely related to the Lefschetz zeta function $\zeta_{L}$, which we shall use henceforth. Baladi and Ruelle [1] have shown how to replace $z$ in the MilnorThurston formula by $Z=\left(z_{1}, \ldots, z_{N}\right)$, where the interval of definition of $f$ is cut into subintervals with different weights $z_{i}$. We shall here use a further extension of the formula $\zeta_{L}(Z)=D(Z)$, where $f$ is allowed to be multivalued. The inverse $f^{-1}$ of $f$ is again multivalued piecewise monotone; it is associated with a zeta function $\zeta_{L}^{\prime}(Z)$. There is a natural relation (functional equation)

$$
\zeta_{L}(Z) \zeta_{L}^{\prime}(\varepsilon Z)=\zeta_{0}(Z)
$$

where $\varepsilon$ corresponds to some sign changes and $\zeta_{0}(Z)$ counts "exceptional" orbits (generically $\zeta_{0}(Z)=1$ ). The analytic properties of $\zeta_{L}(Z)$ are related, via the kneading determinant $D(Z)$, to the spectral properties of a transfer operator $\mathscr{M}_{Z}$. The spectral properties needed here are a refinement of those proved in Ruelle [4]. Using these properties one shows that $\zeta_{L}$ is meromorphic in a certain domain, with poles only if 1 is an eigenvalue of $\mathscr{M}_{Z}$. Let $\mathscr{M}_{Z}^{\prime}$ denote the transfer operator corresponding to $f^{-1}$; using the functional equation one shows that $\zeta_{L}$ can vanish only if 1 is an eigenvalue of $\mathscr{M}_{Z}^{\prime}$.

In what follows we shall write $\zeta$ instead of $\zeta_{L}$, and use a family $\left(\psi_{\omega}\right)$ of monotone maps, instead of the multivalued map $f^{-1}$. Warning: If the $\psi_{\omega}$ are the branches 
of the inverse of a function $f$, the zeta function of [1] is here denoted by $1 / \zeta(\varepsilon Z)$, and the kneading determinant by $\widehat{D}(Z)$ (see Sect. 1.10) rather than $D(Z)$.

I wish to thank Viviane Baladi who carefully read the present paper, and suggested a number of improvements which have been incorporated in the manuscript.

\section{Definitions and Statement of Results}

1.1. Lefschetz Numbers. We shall use the notation

$$
\operatorname{sgn} x=\left\{\begin{array}{ll}
+1 & \text { if } x>0 \\
0 & \text { if } x=0 \\
-1 & \text { if } x<0
\end{array} \quad \text { del } x=\left\{\begin{array}{ll}
+1 & \text { if } x=0 \\
0 & \text { if } x \neq 0
\end{array} .\right.\right.
$$

Let $a<b$, and $\psi:(a, b) \mapsto \mathbb{R}$ be continuous and strictly monotone. We let $\varepsilon=+1$ if $\psi$ is increasing, -1 if $\psi$ is decreasing, and we define the Lefschetz number $L(\psi)$ by

$$
\begin{aligned}
L(\psi) & =L_{1}(\psi)+L_{0}(\psi), \\
L_{1}(\psi) & =\frac{1}{2}[\operatorname{sgn}(\bar{\psi}(a)-a)-\operatorname{sgn}(\bar{\psi}(b)-b)], \\
L_{0}(\psi) & =\frac{\varepsilon}{2}[\operatorname{del}(\bar{\psi}(a)-a)+\operatorname{del}(\bar{\psi}(b)-b)],
\end{aligned}
$$

where $\bar{\psi}$ denotes the extension of $\psi$ by continuity to $[a, b]$, so that $\bar{\psi}(a)=$ $\lim _{x \downarrow a} \psi(a), \bar{\psi}(b)=\lim _{x \uparrow b} \psi(x)$.

Therefore, when $\varepsilon=+1$ we have

$$
L(\psi)=\left\{\begin{array}{ll}
1 & \text { if } \bar{\psi}(a) \geqq a \text { and } \bar{\psi}(b) \leqq b \\
-1 & \text { if } \bar{\psi}(a)<a \text { and } \bar{\psi}(b)>b
\end{array},\right.
$$

when $\varepsilon=-1$ we have

$$
L(\psi)=1 \quad \text { if } \bar{\psi}(a)>a \text { and } \bar{\psi}(b)<b,
$$

and in all other cases we have

$$
L(\psi)=0 .
$$

Let $\operatorname{Fix} \psi=\{x \in(a, b): \psi x=x\}$. If Fix $\psi$ is finite and $x \in \operatorname{Fix} \psi$ we write

$$
L(x, \psi)=\frac{1}{2}\left[\lim _{y \uparrow x} \operatorname{sgn}(\psi(y)-y)-\lim _{y \downarrow x} \operatorname{sgn}(\psi(y)-y)\right] .
$$

Lemma (Properties of Lefschetz numbers). (a) If a $C^{0}$-small perturbation $\tilde{\psi}$ of $\psi$ shrinks the range (i.e., $\tilde{\psi}(a, b) \subset \psi(a, b))$ then it preserves the Lefschetz number (i.e., $L(\tilde{\psi})=L(\psi)$ ).

(b) Consider $\psi^{-1}$ defined on the open interval $\psi(a, b)$, then

$$
\begin{aligned}
& L_{1}\left(\psi^{-1}\right)=-\varepsilon L_{1}(\psi), \\
& L_{0}\left(\psi^{-1}\right)=L_{0}(\psi) .
\end{aligned}
$$

(c) Let Fix $\psi$ be finite and $\tilde{\psi}(a) \neq a, \tilde{\psi}(b) \neq b$. Then

$$
L(\psi)=\sum_{x \in \operatorname{Fix} \psi} L(x, \psi) .
$$


Part (a) of the lemma follows from the list given above of cases when $L(\psi)=$ $1,-1$, or 0 . Part (b) results directly from the definitions. To prove (c) notice that by assumption

$$
\begin{aligned}
L(\psi) & =L_{1}(\psi)=\frac{1}{2}[\operatorname{sgn}(\bar{\psi}(a)-a)-\operatorname{sgn}(\bar{\psi}(b)-b)] \\
& =\frac{1}{2}\left[\lim _{y \downarrow a} \operatorname{sgn}(\psi(y)-y)-\lim _{y \uparrow b} \operatorname{sgn}(\psi(y)-y)\right]=\sum_{x \in \mathrm{F}_{1 X} \psi} L(x, \psi) .
\end{aligned}
$$

This concludes the proof.

1.2. Zeta Functions. Let $\left(J_{\omega}\right),\left(\psi_{\omega}\right),\left(\varepsilon_{\omega}\right),\left(z_{\omega}\right)$ be families indexed by $\omega \in\{1, \ldots, N\}$, where $J_{\omega}=\left(u_{\omega}, v_{\omega}\right)$ is a nonempty bounded interval of $\mathbb{R} ; \psi_{\omega}: J_{\omega} \rightarrow \mathbb{R}$ is a strictly monotone continuous map; $\varepsilon_{\omega}=+1$ or -1 depending on whether $\psi_{\omega}$ is increasing or decreasing; and $z_{\omega} \in \mathbb{C}$. We write $Z=\left(z_{\omega}\right), \varepsilon Z=\left(\varepsilon_{\omega} z_{\omega}\right)$.

It will be convenient to assume henceforth that all $J_{\omega}$ and $\psi_{\omega} J_{\omega}$ are contained in $(-1,+1)$; this is no restriction of generality since $\mathbb{R}$ can be mapped homeomorphically on $(-1,+1)$.

If $m \geqq 1$ and $\omega=\left(\omega_{1}, \ldots, \omega_{m}\right) \in\{1, \ldots, N\}^{m}$, we write $|\omega|=m, \varepsilon(\omega)=$ $\prod_{k=1}^{m} \varepsilon_{\omega_{k}}, Z(\omega)=\prod_{k=1}^{m} z_{\omega_{k}}$. We also let $\psi_{\omega}: J_{\omega} \rightarrow \mathbb{R}$ be defined by $\psi_{\omega}=\psi_{\omega_{m}}$ 。 $\cdots \circ \psi_{\omega_{1}}$, on

$$
J_{\omega}=J_{\omega_{1}} \cap \psi_{\omega_{1}}^{-1}\left(J_{\omega_{2}} \cap \psi_{\omega_{2}}^{-1}\left(\cdots \psi_{\omega_{m}-1}^{-1} J_{\omega_{m}} \cdots\right)\right)
$$

If $J_{\omega} \neq \emptyset$, we write $J_{\omega}=\left(u_{\omega}, v_{\omega}\right)$.

The Lefschetz zeta function (associated with the data $\left.\left(J_{\omega}\right),\left(\psi_{\omega}\right)\right)$ is the formal power series

$$
\zeta(Z)=\exp \sum_{\omega} \frac{1}{|\omega|} L\left(\psi_{\omega}\right) Z(\omega)
$$

where the sum is restricted to those $\omega$ for which $J_{\omega} \neq \emptyset$ (or one defines $L\left(\psi_{\omega}\right)=0$ when $J_{\omega}=\emptyset$ ). One can write a product formula for $\zeta(Z)$ (see Appendix A) and check that $\zeta(Z), 1 / \zeta(Z) \in \mathbb{Z}\left[\left[z_{1}, \ldots, z_{N}\right]\right]$ (Lemma A.2). The zeta function associated with the data $\left(\psi_{\omega} J_{\omega}\right),\left(\psi_{\omega}^{-1}\right)$ is

$$
\zeta^{\prime}(Z)=\exp \sum_{\omega} \frac{1}{|\omega|} L\left(\psi_{\omega}^{-1}\right) Z(\omega)
$$

and we write

$$
\widehat{\zeta}(Z)=\zeta^{\prime}(\varepsilon Z)
$$

We shall also need the function

$$
\begin{aligned}
\zeta_{0}(Z) & =\exp \sum_{\omega} \frac{1}{|\omega|} L_{0}\left(\psi_{\omega}\right)(1+\varepsilon(\omega)) Z(\omega) \\
& =\exp \sum_{\omega: \varepsilon(\omega)=1} \frac{1}{|\omega|}\left[\operatorname{del}\left(\bar{\psi}_{\omega}\left(u_{\omega}\right)-u_{\omega}\right)+\operatorname{del}\left(\bar{\psi}_{\omega}\left(v_{\omega}\right)-v_{\omega}\right)\right] Z(\omega)
\end{aligned}
$$

1.3. Transfer Operators and Kneading Determinant. We introduce the (generalized) transfer operator $\mathscr{M}=\mathscr{M}_{Z}$, the formal adjoint $\mathscr{M}^{\prime}=\mathscr{M}_{Z}^{\prime}$, and the associated 
operator $\widehat{\mathscr{M}}$ such that

$$
\begin{aligned}
\mathscr{M} \Phi(x) & =\sum_{\omega} z_{\omega} \chi_{\omega}(x) \Phi\left(\psi_{\omega} x\right), \\
\mathscr{M}^{\prime} \Phi(x) & =\sum_{\omega} z_{\omega} \chi_{\omega}^{\prime}(x) \Phi\left(\psi_{\omega}^{-1} x\right), \\
\widehat{\mathscr{M}} & =\widehat{\mathscr{M}}_{Z}=\mathscr{M}_{\varepsilon Z}^{\prime},
\end{aligned}
$$

where $\chi_{\omega}$ is the characteristic function of $J_{\omega}$ and $\chi_{\omega}^{\prime}$ the characteristic function of $\psi_{\omega} J_{\omega}$. These operators act on the Banach space $\mathscr{B}$ of functions of bounded variation $\mathbb{R} \rightarrow \mathbb{C}$. It is also convenient to consider them as acting on the Banach space of bounded functions $\mathbb{R} \rightarrow \mathbb{C}$ (with the uniform norm $\|\cdot\|_{0}$ ).

We define $R=R(Z), R^{\prime}=R^{\prime}(Z)$ and $\widehat{R}$ by

$$
\begin{aligned}
R & =\lim _{m \rightarrow \infty}\left(\left\|\mathscr{M}^{m}\right\|_{0}\right)^{1 / m}, \\
R^{\prime} & =\lim _{m \rightarrow \infty}\left(\left\|\mathscr{M}^{\prime m}\right\|_{0}\right)^{1 / m}, \\
\widehat{R} & =\widehat{R}(Z)=R^{\prime}(\varepsilon Z) .
\end{aligned}
$$

The submultiplicativity of $m \mapsto\left\|\mathscr{M}^{m}\right\|_{0},\left\|\mathscr{M}^{\prime m}\right\|_{0}$ guarantees the existence of the limits; $R, R^{\prime}$ and $\widehat{R}$ are in fact the spectral radii of $\mathscr{M}, \mathscr{M}^{\prime}$ and $\widehat{\mathscr{M}}$ acting on bounded functions $\mathbb{R} \rightarrow \mathbb{C}$. In general $R \neq \widehat{R}$.

Let $\left\{a_{1}, \ldots, a_{L}\right\}$ contain the set of all endpoints $u_{\omega}, v_{\omega}$ of the intervals $J_{\omega}$, and assume that $a_{1}<\cdots<a_{L}$. We define $\alpha_{i} \in \mathscr{B}$ by

$$
\alpha_{i}(x)=\operatorname{sgn}\left(x-a_{l}\right)
$$

for $i=1, \ldots, L$, and write

$$
\begin{aligned}
& D_{i j}^{(m)+}=\lim _{x \downarrow a_{l}} \sum_{\omega: u_{\omega}=a_{l}} z_{\omega} \cdot\left[\left(\mathscr{M}^{m-1} \alpha_{j}\right)\left(\psi_{\omega} x\right)\right], \\
& D_{i j}^{(m)-}=\lim _{x \uparrow a_{i}} \sum_{\omega: v_{\omega}=a_{l}} z_{\omega} \cdot\left[\left(\mathscr{M}^{m-1} \alpha_{j}\right)\left(\psi_{\omega} x\right)\right] .
\end{aligned}
$$

The elements of the $L \times L$ kneading matrix $\left[D_{i j}\right]$ are then defined by

$$
D_{i j}(Z)=\delta_{i j}+\sum_{m=1}^{\infty} \frac{1}{2}\left[D_{l j}^{(m)+}-D_{l j}^{(m)-}\right]
$$

(this is an extension of the concept of kneading matrix introduced by Milnor and Thurston [2]). The determinant

$$
D(Z)=\operatorname{det}\left[D_{i j}(Z)\right] \in \mathbb{Q} \llbracket z_{1}, \ldots, z_{N} \rrbracket
$$

is called kneading determinant.

\subsection{Theorem A. We have identically}

$$
\zeta(Z)=D(Z) .
$$

This will be proved using a homotopy argument similar to the one used originally by Milnor and Thurston [2], and then by Baladi and Ruelle [1] in an analogous situation. This means that (for fixed families $\left(J_{\omega}\right),\left(\varepsilon_{\omega}\right),\left(z_{\omega}\right)$ ) first the formula $\zeta=D$ 
is checked for a special choice $\psi^{0}$ of $\psi$. Then, for a suitable one-parameter family $\left(\psi^{\lambda}\right)$ with $\psi^{1}=\psi$, one verifies that $\zeta$ and $D$ are multiplied by the same factor at each bifurcation. The proof presented here is similar to that of [1], but with significant differences; we defer it to Appendix A.

1.5. Theorem B. (a) The spectral radius of $\mathscr{M}$, acting on $\mathscr{B}$, is $\leqq \max (R, \widehat{R})$.

(b) The essential spectral radius of $\mathscr{M}$ is $\leqq \widehat{R}$.

This is closely related to the results of Ruelle [4] but, again, with significant differences. In Appendix B we give an improved version of the theorem of [4], which will yield Theorem B as a special case.

1.6. Theorem C. (a) We have identically $\zeta(Z) \cdot \widehat{\zeta}(Z)=\zeta_{0}(Z)$.

(b) $\zeta(Z)$ is holomorphic when $R(Z)<1$.

(c) $\zeta(Z)$ is meromorphic when $\widehat{R}(Z)<1$, with poles only when $1 \in$ spectrum $\mathscr{M}_{Z}$.

(d) $\zeta_{0}(Z)$ is holomorphic when $\min \{R(Z), \widehat{R}(Z)\}<1$.

This is proved in Sect. 2, and some strengthening of the theorem is provided by the four remarks below.

1.7. Remark. Sharpening of Theorem C. Define

$$
\mathscr{B}_{\infty}=\{A \in \mathscr{B}:\{x: A(x) \neq 0\} \text { is countable }\}
$$

and let

$$
\mathscr{B}^{\#}=\mathscr{B} / \mathscr{B}_{\infty}
$$

be the quotient Banach space. If $\Phi \in \mathscr{B}$, we may define $\Phi^{\#}$ by

$$
\Phi^{\#}(x)=\frac{1}{2}\left[\lim _{y \downarrow x} \Phi(y)+\lim _{y \uparrow x} \Phi(y)\right] .
$$

We have then the properties

$$
\begin{aligned}
\Phi & =\Phi^{\#}+\Phi_{\infty}, \quad \Phi_{\infty} \in \mathscr{B}_{\infty}, \\
\Phi^{\#}(x) & =\frac{1}{2}\left[\lim _{y \downarrow x} \Phi^{\#}(y)+\lim _{y \uparrow x} \Phi^{\#}(y)\right] .
\end{aligned}
$$

If $\|[\Phi]\|^{\#}$ denotes the norm of the class of $\Phi$ in $\mathscr{B} / \mathscr{B}_{\infty}=\mathscr{B}^{\#}$ we have

$$
\|[\Phi]\|^{\#}=\left\|\Phi^{\#}\right\|_{\mathscr{B}} .
$$

Using $\|\cdot\|_{0}^{\#}$ to denote the "sup norm up to a countable set" we see that $\|\cdot\|_{0}^{\#}$ is defined on $\mathscr{B}^{\#}$ and that

$$
\|[\Phi]\|_{0}^{\#}=\left\|\Phi^{\#}\right\|_{0} .
$$

Since $\mathscr{B}_{\infty}$ is stable under $\mathscr{M}, \widehat{\mathscr{M}}$ we may, by going to the quotient, define operators $\mathscr{M}^{\#}, \widehat{\mathscr{M}^{\#}}$ on $\mathscr{B}^{\#}$. We also use the notation $\mathscr{M}^{\#}, \widehat{\mathscr{M}}^{\#}$ for $\mathscr{M}$. $\widehat{\mathscr{M}}$ acting on bounded fucntions up to a countable set. We may then write

$$
\begin{aligned}
& R^{\#}=R^{\#}(Z)=\lim _{m \rightarrow \infty}\left(\left\|\mathscr{M}^{\# m}\right\|_{0}^{\#}\right)^{1 / m}, \\
& \widehat{R}^{\#}=\widehat{R}^{\#}(Z)=\lim _{m \rightarrow \infty}\left(\left\|\widehat{\mathscr{M}}^{\# m}\right\|_{0}^{\#}\right)^{1 / m} .
\end{aligned}
$$


The point of the above definitions is that in defining the kneading matrix $\left[D_{l j}\right]$ we may neglect countable sets, i.e., use the operator $\mathscr{M}^{\#}$ instead of $\mathscr{M}$. As a consequence of this we may replace $\mathscr{M}, \widehat{\mathscr{M}}, R, \widehat{R}$ by $\mathscr{M}^{\#}, \widehat{\mathscr{M}}^{\#}, R^{\#}, \widehat{R}^{\#}$ in the statement of Theorem C (b), (c), (d). We shall not give an explicit demonstration of the results thus obtained, but note that they follow by inspection of the proofs in Appendix B (\#-version of Theorem B. 1) and Sect. 2. The basic fact is that the continuous linear functionals on $\mathscr{B}$ defined by $\Phi \mapsto \lim _{x \downarrow a}, \lim _{x \uparrow a} \Phi(x)$ yield continuous linear functionals on $\mathscr{B}^{\#}$ (while $\Phi \mapsto \Phi(a)$ is not defined on $\mathscr{B}^{\#}$ ).

The set $Z_{m}=X_{m} \cup Y_{m}$, with $X_{m}=\left\{u_{\omega}, v_{\omega}:|\omega|=m\right\}, Y_{m}=\left\{x: \psi_{\omega}(x)=\psi_{\omega^{\prime}}(x)\right.$ with $|\omega|=\left|\omega^{\prime}\right|=m$ and $\left.\varepsilon\left(\omega^{\prime}\right)=-\varepsilon(\omega)\right\}$ is finite. Given $x \notin Z_{m}$ there is $\delta>0$ such that for each bounded $\Phi$ we may construct $\Phi_{\varepsilon}$ with $\left\|\Phi_{\varepsilon}\right\|_{0}=\|\Phi\|_{0}$, and $\Phi_{\varepsilon}\left(\psi_{\omega} y\right)=$ $\varepsilon(\omega) \Phi\left(\psi_{\omega} y\right)$ when $|y-x|<\delta$ and $|\omega|=m$. We have then

$$
\left(\mathscr{M}_{\varepsilon Z}^{m} \Phi\right)(y)=\left(\mathscr{M}_{Z}^{m} \Phi_{\varepsilon}\right)(y) \text { if }|y-x|<\delta,
$$

hence

$$
\left\|\mathscr{M}_{\varepsilon Z}^{* m}\right\|_{0}^{\#} \leqq\left\|\mathscr{M}_{Z}^{\# m}\right\|_{0}^{\#}
$$

hence by symmetry

$$
\left\|\mathscr{M}_{\varepsilon Z}^{\# m}\right\|_{0}^{\#}=\left\|\mathscr{M}_{Z}^{\# m}\right\|_{0}^{\#}
$$

and therefore

$$
R^{\#}(\varepsilon Z)=R^{\#}(Z)
$$

Since $R^{\#}(Z) \leqq R(Z)$ we also have

$$
R^{\#}(Z) \leqq \min \{R(Z), R(\varepsilon Z)\}
$$

Notice that Theorem $\mathrm{B}(\mathrm{b})$ can also be sharpened as follows: the essential spectral radius of $\mathscr{M}$ is $\leqq \widehat{R}^{\#}$. To prove this it suffices to find $\widetilde{K}_{m}$ of finite rank such that

$$
\limsup _{m \rightarrow \infty}\left\|\mathscr{M}^{m}-\widetilde{K}_{m}\right\|^{1 / m} \leqq \widehat{R}^{\#}
$$

We write as above $\Phi=\Phi^{\#}+\Phi_{\infty}$, so that

$$
\left\|\Phi^{\#}\right\|_{\mathscr{B}}=\|[\Phi]\|^{\#} \text { and }\left\|\Phi_{\infty}\right\|_{\mathscr{B}} \leqq\|\Phi\|_{\mathscr{B}}
$$

Let $\chi$ be the characteristic function of $\bigcup_{\omega}\left\{\bar{\psi}_{\omega} u_{\omega}, \bar{\psi}_{\omega} v_{\omega}\right\}$ with $|\omega|=m$. The map $E: \Phi \mapsto \chi \Phi$ is of finite rank, and so is $K_{m}^{\prime}=\mathscr{M}^{m} E$. Note that when $y \notin \bigcup_{\omega}\left\{\bar{\psi}_{\omega} u_{\omega}, \bar{\psi}_{\omega} v_{\omega}\right\}$ and $\Psi=\Psi^{\#}$, we have $\left(\mathscr{M}^{\prime m} \Psi\right)(y)=\left(\mathscr{M}^{\prime m} \Psi\right)^{\#}(y)$. We may now write

$$
\begin{aligned}
\operatorname{Var}\left(\mathscr{M}^{m}-K_{m}^{\prime}\right) \Phi_{\infty} & =\operatorname{Var} \mathscr{M}^{m}\left(\Phi_{\infty}-\chi \Phi_{\infty}\right)=2 \sum_{x}\left|\mathscr{M}^{m}\left(\Phi_{\infty}-\chi \Phi_{\infty}\right)(x)\right| \\
& =2 \sup \left|\sum_{x} \Psi(x)\left[\mathscr{M}^{m}\left(\Phi_{\infty}-\chi \Phi_{\infty}\right)\right](x)\right|
\end{aligned}
$$


(where the sup is over $\Psi$ such that $\|\Psi\|_{0}=1$ and $\Psi=\Psi^{\#}$ )

$$
\begin{aligned}
& =2 \sup \left|\sum_{y}\left(\mathscr{M}^{\prime m} \Psi\right)(y) \cdot\left(\Phi_{\infty}-\chi \Phi_{\infty}\right)(y)\right| \\
& =2 \sup \left|\sum_{y}\left(\mathscr{M}^{\prime m} \Psi\right)^{\#}(y) \cdot\left(\Phi_{\infty}-\chi \Phi_{\infty}\right)(y)\right| \\
& \leqq\left\|\left(\mathscr{M}^{\prime \#}\right)^{m}\right\|_{0}\left\|\Phi_{\infty}\right\|_{\mathscr{S}} .
\end{aligned}
$$

In conclusion if $\varepsilon>0$ we have

$$
\left\|\left(\mathscr{M}^{m}-K_{m}^{\prime}\right) \Phi_{\infty}\right\|_{\mathscr{B}} \leqq \operatorname{const}\left(\widehat{R}^{\#}+\varepsilon\right)^{m}\|\Phi\|_{\mathscr{B}} .
$$

By the proof of Theorem B (\#-version) there is $K_{m}^{\#}$ of finite rank on $\mathscr{B}^{\#}$ such that

$$
\left\|\mathscr{M}^{\# m}-K_{m}^{\#}\right\|^{\#} \leqq \operatorname{const}\left(\widehat{R}^{\#}+\varepsilon\right)^{m} .
$$

We choose $K_{m}$ of finite rank on $\mathscr{B}$ such that $K_{m}$ induces $K_{m}^{\#}$ on $\mathscr{B}^{\#}$ and $K_{m} \Phi=\left(K_{m} \Phi\right)^{\#}$. There is also $K_{m}^{\prime \prime}$ of finite rank such that

$$
\mathscr{M}^{m} \Phi^{\#}-K_{m}^{\prime \prime} \Phi^{\#}=\left(\mathscr{M}^{m} \Phi^{\#}\right)^{\#}
$$

Therefore

$$
\mathscr{M}^{m} \Phi^{\#}-K_{m} \Phi^{\#}-K_{m}^{\prime \prime} \Phi^{\#}=\left(\mathscr{M}^{m} \Phi^{\#}-K_{m} \Phi^{\#}\right)^{\#}
$$

and

$$
\left\|\left(\mathscr{M}^{m}-K_{m}-K_{m}^{\prime \prime}\right) \Phi^{\#}\right\|_{\mathscr{B}}=\left\|\left(\mathscr{M}^{\# m}-K_{m}^{\#}\right)[\Phi]\right\|^{\#} \leqq \operatorname{const}\left(\widehat{R}^{\#}+\varepsilon\right)^{m}\|\Phi\|_{\mathscr{B}} .
$$

Defining now $\widetilde{K}_{m} \Phi=\left(K_{m}+K_{m}^{\prime \prime}\right) \Phi^{\#}+K_{m}^{\prime} \Phi_{\infty}$ we obtain

$$
\left\|\left(\mathscr{M}^{m}-\widetilde{K}_{m}\right) \Phi\right\|_{\mathscr{B}} \leqq \operatorname{const}\left(\widehat{R}^{\#}+\varepsilon\right)^{m}\|\Phi\|_{\mathscr{B}},
$$

and therefore $(*)$ holds.

One can also show that the spectral radius of $\mathscr{M}^{\#}$ is $\geqq \widehat{R}^{\#}$ (this will not be used).

If $\Phi \in \mathscr{B}_{\infty}$ we have

$$
\begin{aligned}
\operatorname{Var} \mathscr{M}^{m} \Phi & =2 \sum_{x}\left|\left(\mathscr{M}^{m} \Phi\right)(x)\right|=2 \sup _{\Psi:\|\Psi\|_{0}=1}\left|\sum_{x} \Psi(x) \cdot\left(\mathscr{M}^{m} \Phi\right)(x)\right| \\
& =2 \sup _{\Psi}\left|\sum_{y}\left(\mathscr{M}^{\prime m} \Psi\right)(y) \cdot \Phi(y)\right| \leqq\left\|\mathscr{M}^{\prime m}\right\|_{0} \cdot \operatorname{Var} \Phi
\end{aligned}
$$

so that the spectral radius of $\mathscr{M}_{Z} \mid \mathscr{B}_{\infty}$ is $\leqq \widehat{R}(\varepsilon Z)$. In particular $\mathscr{M}_{Z}$ and $\mathscr{M}_{Z}^{\#}$ have the same eigenvalues $\lambda$ with the same multiplicity when $|\lambda|>\max (\widehat{R}(Z), \widehat{R}(\varepsilon Z))$.

1.8. Remark. Further properties of $\zeta_{0}$. The proof of Lemma 2.4 below shows that if $\zeta_{0}(Z)=0$ and $\widehat{R}^{\#}(Z)<1$, then 1 belongs to the spectrum of $\mathscr{M}_{Z} \mid \mathscr{B}_{\infty}$ or $\mathscr{M}_{\varepsilon Z} \mid \mathscr{B}_{\infty}$. Similarly, if $\zeta_{0}(Z)=0$ and $R^{\#}(Z)<1$, then 1 belongs to the spectrum of $\widehat{\mathscr{M}}_{Z} \mid \mathscr{B}_{\infty}$ or $\widehat{\mathscr{M}}_{\varepsilon Z} \mid \mathscr{B}_{\infty}$. 
The following condition is generically satisfied.

Condition $G$. For all $m \geqq 1$ and $\omega=\left(\omega_{1}, \ldots, \omega_{m}\right)$ with $\varepsilon(\omega)=1$, we have

$$
\begin{array}{ll}
\bar{\psi}_{\omega} u_{\omega_{1}} \neq u_{\omega_{1}} & \text { if } \bar{\psi}_{\omega} u_{\omega_{1}} \text { is defined, } \\
\bar{\psi}_{\omega} v_{\omega_{1}} \neq v_{\omega_{1}} & \text { if } \bar{\psi}_{\omega} v_{\omega_{1}} \text { is defined. }
\end{array}
$$

It is clear from the definition of $\zeta_{0}$ that if Condition $\mathrm{G}$ holds, then $\zeta_{0}=1$ identically.

1.9. Remark. Poles of $D(z Z)$. Let $Z$ be fixed. The function $z \mapsto D(z Z)$ is meromorphic when $|z| \widehat{R}^{\#}(Z)<1$, and clearly can have a pole at $\lambda^{-1}$ only if $\lambda$ is an eigenvalue of $\mathscr{M}^{\#}=\mathscr{M}_{Z}^{\#}$. Let

$$
\mathscr{D}=\left\{z:|z|<\widehat{R}^{\#}(Z)^{-1} \text { and } z^{-1} \text { is not an eigenvalue of } \mathscr{M}_{\varepsilon Z} \mid B_{\infty}\right\} .
$$

In particular (see the end of Remark 1.7),

$$
\left\{z:|z|<\widehat{R}(Z)^{-1}\right\} \subset \mathscr{D} .
$$

We shall show that the function $z \mapsto D(z Z)$ does not vanish in $\mathscr{D}$, and has a pole of order $m$ at $\lambda^{-1}$ precisely if $\lambda$ is an eigenvalue of order $m$ of $\mathscr{M}^{\#}$.

The proof will be in several steps.

(i) Let us define

$$
A=\left\{a_{1}, \ldots, a_{L}\right\} \cup\left\{\psi_{\omega}^{-1} a_{\mathbf{i}}:|\omega| \geqq 1,1 \leqq i \leqq L\right\},
$$

$\mathscr{B}_{A}^{\#}=\left\{[\Phi] \in \mathscr{B}^{\#}:\right.$ the derivative of $\Phi$ is an atomic measure carried by $\left.A\right\}$.

Then the generalized eigenspace of $\mathscr{M}^{\#}$ corresponding to any eigenvalue $\lambda$ with $|\lambda|>\widehat{R} \#(Z)$ is contained in $\mathscr{B}_{A}^{\#}$.

We may extend the linear operator $\mathscr{M}_{Z}$ from bounded functions to measures by letting

$$
\left(\mathscr{M}_{Z} \mu\right)(d x)=\sum_{\omega} z_{\omega} \chi_{\omega}(X) \cdot\left(\psi_{\omega}^{-1} \mu\right)(d x)
$$

(where $\psi_{\omega}^{-1} \mu$ is the image of $\mu$ by $\psi_{\omega}^{-1}$ ). We shall write

$$
(\Psi, \mu)=\int \mu(d x) \Psi(x)
$$

if $\Psi$ is a continuous function. If $\Phi$ is of bounded variation, we denote by $\partial \Phi$ its derivative, which is a bounded measure. (If $\Phi \in \mathscr{B}_{\infty}$, then $\partial \Phi=0$. Therefore $\partial \Phi$ only depends on the class $[\Phi] \in \mathscr{B}^{\#}$.) We also let $\mathscr{P}$ be the projection on measures $\mu$ such that $|\mu|(A)=0$ (i.e., $\mathscr{P}$ "erases" the mass carried by $A$ ). If $X: \mathbb{R} \mapsto\{0,1\}$ is 0 on $\left\{a_{1}, \ldots, a_{L}\right\}$ and 1 elsewhere, we have

$$
\mathscr{P} \partial \mathscr{M}_{Z} \Phi=X \mathscr{M}_{\varepsilon Z} \mathscr{P} \partial \Phi \text {. }
$$

When $[\mathscr{M} \Phi]=\lambda[\Phi]\left(\bmod \mathscr{B}_{A}^{\#}\right)$ we have thus

$$
\begin{aligned}
(\Psi, \mathscr{P} \partial \Phi) & =\lambda^{-m}\left(\Psi, \mathscr{P} \partial \mathscr{M}_{Z}^{m} \Phi\right)=\lambda^{-m}\left(\Psi,\left(X \mathscr{M}_{Z}\right)^{m} \mathscr{P} \partial \Phi\right) \\
& =\lambda^{-m}\left(\left(\widehat{\mathscr{M}}_{\varepsilon Z}^{m} X\right)^{m} \Psi, \mathscr{P} \partial \Phi\right)
\end{aligned}
$$


If $|\lambda|>\widehat{R} \#(Z)$, the right-hand side must vanish, so that $\mathscr{P} \partial \Phi=0$, i.e., $[\Phi] \in \mathscr{B}_{A}^{\#}$. By induction we see that if $\left[(\mathscr{M}-\lambda)^{k} \Phi\right]=0$, i.e., if $[\Phi]$ is in the generalized eigenspace of $\mathscr{M}^{\#}$ corresponding to $\lambda$, we have $[\Phi] \in \mathscr{B}_{A}^{\#}$.

(ii) Let $\lambda^{-1} \in \mathscr{D}$ and suppose that (with $\alpha_{i}$ defined in Sect. 1.3)

Then if

$$
\begin{aligned}
& \left(1-\lambda^{-1} \mathscr{M}^{\#}\right) \Omega=0, \\
& \left(1-\lambda^{-1} \mathscr{M}^{\#}\right) \gamma_{j}=\alpha_{j} \quad \text { for } j=1, \ldots, L .
\end{aligned}
$$

$$
\left(1-\lambda^{-1} \mathscr{M}_{\varepsilon Z}\right) \partial\left(\Omega+\sum_{j=1}^{L} c_{j} \gamma_{j}\right)
$$

has no mass at $a_{1}, \ldots, a_{L}$ we have $\Omega=0$ and $c_{1}=\cdots=c_{L}=0$.

Let us write

$$
\Phi=\sum c_{j} \alpha_{j}, \quad \Psi=\Omega+\sum c_{j} \gamma_{j} .
$$

Then $\left(1-\lambda^{-1} \mathscr{M}_{Z}^{\#}\right) \Psi=\Phi$; in particular $\mathscr{M}_{Z}^{\#} \Psi=\lambda \Psi\left(\bmod \mathscr{B}_{A}^{\#}\right)$ which implies $\Psi \in \mathscr{B}_{A}^{\#}$ as we have seen in (i). Furthermore $\left(1-\lambda^{-1} \mathscr{M}_{\varepsilon Z}\right) \partial \Psi$ has no mass outside of $a_{1}, \ldots, a_{L}$, so that by assumption

$$
\left(1-\lambda^{-1} \mathscr{M}_{\varepsilon Z}\right) \partial \Psi=0 .
$$

Since $\Psi \in \mathscr{B}_{A}$, this is equivalent to

$$
\left(1-\lambda^{-1} \mathscr{M}_{\varepsilon Z}\right) \widetilde{\Psi}=0
$$

with $\widetilde{\Psi} \in \mathscr{B}_{\infty}$ such that $\widetilde{\Psi}(x)=(\partial \Psi)(\{x\})$, and the assumption $\lambda^{-1} \in \mathscr{D}$ implies $\widetilde{\Psi}=0$, i.e., $\partial \Psi=0$, i.e., $\Psi=$ constant. Therefore $\Phi$ tends to the constant $\Psi$ at $\pm \infty$, but since $\Phi(-\infty)=-\Phi(\infty)$, we obtain $\Psi=0$. Therefore $\Phi=0$, so that $c_{1}=\cdots=c_{L}=0$, and finally also $\Omega=0$.

(iii) If $\lambda^{-1} \in \mathscr{D}$ and $\lambda$ is not an eigenvalue of $\mathscr{M}^{\#}$, then $D\left(\lambda^{-1} Z\right) \neq 0$.

We may write $\gamma_{j}=\left(1-\lambda^{-1} \mathscr{M}^{\#}\right)^{-1} \alpha_{j}$ and define $\Phi=\sum c_{j} \alpha_{j}, \Psi=(1-$ $\left.\lambda^{-1} \mathscr{M}_{Z}^{\#}\right)^{-1} \Phi=\sum c_{j} \gamma_{j}$. Suppose there is a linear relation

$$
\sum c_{j} D_{l j}\left(\lambda^{-1} Z\right)=0
$$

between the columns of $\left(D_{i j}\right)$, i.e.,

$$
c_{i}+\frac{1}{2} \lim _{x \downarrow a_{i}} \sum_{\omega: u_{\omega}=a_{l}} \lambda^{-1} z_{\omega} \Psi\left(\psi_{\omega} x\right)-\frac{1}{2} \lim _{x \uparrow a_{l}} \sum_{\omega: v_{\omega}=a_{l}} \lambda^{-1} z_{\omega} \Psi\left(\psi_{\omega} x\right)=0 .
$$

This may be rewritten as

$$
\beta_{i}(\Phi)+\beta_{i}\left(\lambda^{-1} \mathscr{M}_{Z} \Psi\right)-\text { correction }=0,
$$

where the correction corresponds to those terms $\pm \frac{1}{2} \lim _{x \rightarrow a_{t}} \lambda^{-1} z_{\omega} \Psi\left(\psi_{\omega} x\right)$ such that $a_{i} \varepsilon J_{\omega}$. Equivalently we may write

or

$$
\text { mass at } a_{i} \text { of }\left(\partial \Phi+\partial \lambda^{-1} \mathscr{M}_{Z} \Psi-\lambda^{-1} \mathscr{M}_{\varepsilon Z} \partial \Psi\right)=0
$$

$$
\text { mass at } a_{i} \text { of }\left(\partial \Psi-\lambda^{-1} \mathscr{M}_{\varepsilon} \partial \Psi\right)=0 \text {. }
$$

In view of (ii) we have then $c_{1}=\cdots=c_{L}=0$. Therefore $D\left(\lambda^{-1} Z\right) \neq 0$. 
(iv) If $\lambda^{-1} \in \mathscr{D}$ and $\lambda$ is a simple eigenvalue of $\mathscr{M}^{\#}$, then $\lambda^{-1}$ is a simple pole of $z \mapsto D(z Z)$.

Let $\Omega \neq 0$ be chosen such that $\left(1-\lambda^{-1} \mathscr{M}^{\sharp}\right) \Omega=0$.

First, we show that $\alpha_{1}, \ldots, \alpha_{L}$ cannot all be in the range of $\left(1-\lambda^{-1} \mathscr{M}^{\sharp}\right)$. Otherwise let $\gamma_{1}, \ldots, \gamma_{L}$ be such that

$$
\left(1-\lambda^{-1} \mathscr{M}^{\#}\right) \gamma_{j}=\alpha_{j}
$$

for $j=1, \ldots, L$. In view of (ii) the $L$-dimensional vectors

$$
\text { mass at }\left\{a_{1}, \ldots, a_{L}\right\} \text { of }\left(1-\lambda^{-1} \mathscr{M}_{\varepsilon Z}\right) \partial \gamma_{j}
$$

are linearly independent. Therefore we may take $c_{1}, \ldots, c_{L}$ such that

$$
\text { mass at }\left\{a_{1}, \ldots, a_{L}\right\} \text { of }\left(1-\lambda^{-1} \mathscr{M}_{\varepsilon Z}\right) \partial\left(\Omega+\sum c_{j} \gamma_{j}\right)=0 \text {. }
$$

Using again (ii) yields $\Omega=0$ contrary to assumption. write,

Let us replace $\alpha_{1}, \ldots, \alpha_{L}$ by independent linear combinations $\Phi_{1}, \ldots, \Phi_{L}$ and

so that

$$
\begin{aligned}
& \Psi_{J}(z)=\left(1-z \mathscr{M}^{\#}\right)^{-1} \Phi_{j}, \\
& \Psi_{i j}(z)=\frac{1}{2} \text { mass at } a_{l} \text { of }\left(\partial \Psi_{j}-z \mathscr{M}_{\varepsilon Z} \partial \Psi_{j}\right),
\end{aligned}
$$

$$
D(z Z)=\operatorname{det}\left(\Psi_{l j}(z)\right) .
$$

Since we have shown that $\alpha_{1}, \ldots, \alpha_{L}$ are not all in the range of $\left(1-\lambda^{-1} \mathscr{M}^{\#}\right)^{-1}$, we may assume that $\Psi_{1}(z) \sim(1-z \lambda)^{-1} \Omega$ for $z$ near $\lambda^{-1}$, while $\Psi_{2}(z), \ldots, \Psi_{L}(z)$ are holomorphic at $\lambda^{-1}$. To prove that $\lambda^{-1}$ is a simple pole of $z \mapsto D(z Z)$, it suffices now to show that the vectors

and

$$
\text { mass at }\left\{a_{1}, \ldots, a_{L}\right\} \text { of }\left(1-\lambda^{-1} \mathscr{M}_{\varepsilon Z}\right) \partial \Omega
$$

$$
\text { mass at }\left\{a_{1}, \ldots, a_{L}\right\} \text { of }\left(1-\lambda^{-1} \mathscr{M}_{\varepsilon Z}\right) \partial \Psi_{j}
$$

for $j=2, \ldots, L$ are linearly independent. This again results from (ii).

(v) If $\lambda^{-1} \in \mathscr{D}$ and $\lambda$ is an eigenvalue of order $m$ of $\mathscr{M}^{\#}$, then $\lambda^{-1}$ is a pole of order $m$ of $z \mapsto D(z Z)$.

By extending the index set for $\omega$ from $\{1, \ldots, N\}$ to $\left\{1, \ldots, N^{*}\right\}$ we can obtain small perturbations $\mathscr{M}^{* \#}$ of $\mathscr{M}^{\#}$ and $\mathscr{D}^{*}$ of $\mathscr{D}$ such that $\lambda$ is replaced by $m$ simple eigenvalues $\lambda_{1}^{*}, \ldots, \lambda_{m}^{*}$ contained in a disk $B_{\lambda_{-1}}(\varepsilon) \subset \mathscr{D} \cap \mathscr{D}^{*}$ with small $\varepsilon$. The corresponding $D^{*}(z Z)$ has simple poles and no zero near $\lambda^{-1}$. Since $D^{*}(z Z)$ tends to $D(z Z)$ away from poles it follows that $D(z Z)$ has a pole of order $m$ at $\lambda^{-1}$.

\subsection{Remark. Zeros of $\widehat{D}(z Z)$. Let}

$\mathscr{D}^{*}=\left\{z:|z|<\widehat{R}^{\#}(Z)^{-1}\right.$ and $z^{-1}$ is not an eigenvalue of $\mathscr{M}_{Z} \mid \mathscr{B}_{\infty}$ or $\left.\mathscr{M}_{\varepsilon Z} \mid \mathscr{B}_{\infty}\right\}$.

In particular (see the end of Remark 1.7)

$$
\left\{z:|z|<\min \left\{\widehat{R}(Z)^{-1}, \widehat{R}(\varepsilon Z)^{-1}\right\}\right\} \subset \mathscr{D}^{*} .
$$

Denote by $\widehat{D}$ the kneading determinant associated with $\widehat{\mathscr{M}}$ (so that $\widehat{D}=\widehat{\zeta}$ ). Then, the function $z \mapsto \widehat{D}(z Z)$ is holomorphic in $\mathscr{D}^{*}$ and has a zero of order $m$ at $\lambda^{-1}$ precisely if $\lambda$ is an eigenvalue of order $m$ of $\mathscr{M}^{\#}$ (or equivalently $\mathscr{M}$ ). 
In view of Remark 1.8, the zeros of $\widehat{D}(z Z)$ are the same as the poles of $D(z Z)$, with the same multiplicity. It suffices therefore to apply Remark 1.9. (Since (1 $\left.\lambda^{-1} \mathscr{M}_{Z}\right) \mid \mathscr{B}_{\infty}$ is invertible when $\lambda^{-1} \in \mathscr{D}^{*}$, the multiplicity of $\lambda$ is the same as an eigenvalue of $\mathscr{M}^{\#}$ or $\mathscr{M}$.)

The function $z \mapsto \widehat{D}(z Z)$ in $\mathscr{D}^{*}$ is the natural generalization of the kneading determinant considered by Milnor and Thurston [2], and also in [1].

\section{Proof of Theorem $\mathrm{C}$}

The proof results from the four lemmas below.

2.1. Lemma. We have identically

$$
\zeta(Z) \widehat{\zeta}(Z)=\zeta_{0}(Z)
$$

Using the definitions we obtain

$$
\begin{aligned}
\zeta(Z) \widehat{\zeta}(Z) & =\exp \sum_{\omega} \frac{1}{|\omega|}\left[L\left(\psi_{\omega}\right)+L\left(\psi_{\omega}^{-1}\right) \varepsilon(\omega)\right] Z(\omega) \\
& =\exp \sum_{\omega} \frac{1}{|\omega|}\left[L_{1}\left(\psi_{\omega}\right)+L_{1}\left(\psi_{\omega}^{-1}\right) \varepsilon(\omega)+L_{0}\left(\psi_{\omega}\right)+L_{0}\left(\psi_{\omega}^{-1}\right) \varepsilon(\omega)\right] Z(\omega) \\
& =\exp \sum_{\omega} \frac{1}{|\omega|} L_{0}\left(\psi_{\omega}\right)(1+\varepsilon(\omega)) Z(\omega)=\zeta_{0}(Z),
\end{aligned}
$$

which proves the lemma.

2.2. Lemma. $D_{i j}(Z)$ is holomorphic when $R(Z)<1$.

Suppose that $R\left(Z_{0}\right)<1$, and let $R\left(Z_{0}\right)<\xi<1$. We may then choose $M$ such that

$$
\left\|\mathscr{M}_{Z_{0}}^{M}\right\|_{0}<\xi^{M}
$$

Therefore, for some $\delta>0$, we have

$$
\left\|\mathscr{M}_{Z}^{M}\right\|_{0}<\xi^{M} \quad \text { if }\left|Z-Z_{0}\right|<\delta .
$$

The polynominals $Z \mapsto D_{i j}^{(m) \pm}$ thus satisfy

$$
\left|D_{i j}^{(m) \pm}\right|<C \xi^{m} \quad \text { if }\left|Z-Z_{0}\right|<\delta, m \geqq 0
$$

for some $C>0$. This implies that $D_{i j}(Z)$ is holomorphic for $\left|Z-Z_{0}\right|<\delta$, i.e., $D_{i j}(Z)$ is holomorphic when $R(Z)<1$.

2.3. Lemma. $D_{i j}(Z)$ is meromorphic when $\widehat{R}(Z)<1$, with poles only when $1 \in$ spectrum $\mathscr{M}_{Z}$.

Suppose that $\widehat{R}\left(Z_{0}\right)<1$. We may choose $\xi$ such that $\widehat{R}\left(Z_{0}\right)<\xi<1$ and no eigenvalue of $\mathscr{M}_{Z_{0}}$ has modulus $\xi$ (cf. Theorem $\mathrm{B}(\mathrm{b})$ ). There is then $\delta_{0}>0$ such that, for $\left|Z-Z_{0}\right| \leqq \delta_{0}$, we have $\widehat{R}(Z)<\xi$ and the circle $S=\{\lambda:|\lambda|=\xi\}$ is disjoint from the spectrum of $\mathscr{M}_{Z}$. We then define the projection

$$
P_{Z}=\frac{1}{2 \pi i} \oint_{S} \frac{d \lambda}{\lambda-\mathscr{M}_{Z}} \text {. }
$$


Therefore $P_{Z}$ commutes with $\mathscr{M}_{Z}$, and $1-P_{Z}$ is finite dimensional. We may choose $M$ such that

$$
\left\|P_{Z_{0}} \mathscr{M}_{Z_{0}}^{M}\right\|<\xi^{M}
$$

For some $\delta \in\left(0, \delta_{0}\right)$ we also have

$$
\left\|P_{Z} \mathscr{M}_{Z}^{M}\right\|<\xi^{M} \quad \text { if }\left|Z-Z_{0}\right|<\delta,
$$

hence, for some $C>0$,

$$
\left\|P_{Z} \mathscr{M}_{Z}^{m}\right\|<C \xi^{m} \quad \text { if }\left|Z-Z_{0}\right|<\delta, m \geqq 0 .
$$

Therefore the functions

$$
\begin{aligned}
& \lim _{x \downarrow a_{i}} \sum_{\omega: u_{\omega}=a_{l}} z_{\omega} \cdot\left[\left(P_{Z}\left(1-\mathscr{M}_{Z}\right)^{-1} \alpha_{j}\right)\left(\psi_{\omega} x\right)\right], \\
& \lim _{x \uparrow a_{i}} \sum_{\omega: v_{\omega}=a_{i}} z_{\omega} \cdot\left[\left(P_{Z}\left(1-\mathscr{M}_{Z}\right)^{-1} \alpha_{j}\right)\left(\psi_{\omega} x\right)\right]
\end{aligned}
$$

are holomorphic for $\left|Z-Z_{0}\right|<\delta$. The functions

$$
\begin{aligned}
& \lim _{x \downarrow a_{l}} \sum_{\omega: u_{\omega}=a_{l}} z_{\omega} \cdot\left[\left(\left(1-P_{Z}\right)\left(1-\mathscr{M}_{Z}\right)^{-1} \alpha_{j}\right)\left(\psi_{\omega} x\right)\right], \\
& \lim _{x \uparrow a_{l}} \sum_{\omega: v_{\omega}=a_{J}} z_{\omega} \cdot\left[\left(\left(1-P_{Z}\right)\left(1-\mathscr{M}_{Z}\right)^{-1} \alpha_{j}\right)\left(\psi_{\omega} x\right)\right]
\end{aligned}
$$

are meromorphic for $\left|Z-Z_{0}\right|<\delta$, and in fact holomorphic if $1 \notin$ spectrum $\mathscr{M}_{Z}$. In conclusion $D_{i j}(Z)$ is meromorphic when $\widehat{R}(Z)<1$ and holomorphic unless $1 \in \operatorname{spectrum} \mathscr{M}_{Z}$.

2.4. Lemma. $\zeta_{0}(Z)$ is holomorphic when $\min \{R(Z), \widehat{R}(Z)\}<1$.

Let $A=\left\{a_{1}-, a_{1}+, \ldots, a_{L}-, a_{L}+\right\}$. If $\xi=a_{i} \pm \in A$, we write $|\zeta|=a_{i}$, sign $\zeta= \pm$.

For $\zeta, \eta \in A, m \geqq 1$, we define $T_{\xi \eta}^{(m)}$ to be the sum of the $Z(\omega)$ over all $\omega=\left(\omega_{1}, \ldots, \omega_{m}\right)$ such that

$$
\varepsilon(\omega)=\operatorname{sign} \zeta \cdot \operatorname{sign} \eta
$$

$$
\begin{array}{rll}
\text { either }|\zeta|=u_{\omega_{1}} & \text { and } & \operatorname{sign} \xi=+, \\
\text { or }|\zeta|=v_{\omega_{1}} & \text { and } & \operatorname{sign} \xi=-
\end{array}
$$

$\bar{\psi}_{\omega_{1}}|\xi|$ is in the (open) interval of definition

$$
\text { of } \psi_{\omega_{m}} \circ \cdots \circ \psi_{\omega_{2}} \text {, and } \psi_{\omega_{m}} \circ \cdots \circ \psi_{\omega_{2}} \quad\left(\bar{\psi}_{\omega_{1}}|\xi|\right)=|\eta| \text {. }
$$

Denote by $T=T(Z)$ the matrix with elements

$$
T_{\xi \eta}=\sum_{m \geqq 1} T_{\xi \eta}^{(m)}
$$


We shall now prove that

$$
\begin{aligned}
& \sum_{\omega: \varepsilon(\omega)=1} \frac{Z(\boldsymbol{\omega})}{|\boldsymbol{\omega}|}\left[\operatorname{del}\left(\bar{\psi}_{\omega}\left(u_{\omega}\right)-u_{\omega}\right)+\operatorname{del}\left(\bar{\psi}_{\omega}\left(v_{\omega}\right)-v_{\omega}\right)\right] \\
& =\sum_{n} \frac{1}{n_{\xi_{1}} \cdots \xi_{n}} \sum_{\xi_{1} \xi_{2}} T_{\xi_{2} \xi_{3}} \cdots T_{\xi_{n-1} \xi_{n}} T_{\xi_{n} \xi_{1}} .
\end{aligned}
$$

Consider the symbol $(\omega, \varepsilon)$, where $\omega=\left(\omega_{1}, \ldots, \omega_{m}\right)$ satisfies $\varepsilon(\omega)=1$, and $\varepsilon= \pm 1$. We write $(\boldsymbol{\omega}, \varepsilon) \sim\left(\boldsymbol{\omega}^{\prime}, \varepsilon^{\prime}\right)$ if $\boldsymbol{\omega}^{\prime}=\left(\omega_{k}, \ldots, \omega_{m}, \omega_{1}, \ldots, \omega_{k-1}\right)$ is a circular permutation of $\omega$ and $\varepsilon^{\prime}=\varepsilon \varepsilon_{1} \cdots \varepsilon_{k-1}$. To a nonvanishing term $\operatorname{del}\left(\bar{\psi}_{\omega}\left(u_{\omega}\right)-u_{\omega}\right)$ or $\operatorname{del}\left(\bar{\psi}_{\omega}\left(v_{\omega}\right)-v_{\omega}\right)$ we associate the pair $(\omega,+)$ or $(\omega,-)$ respectively. The left-hand side of the formula $(*)$ may thus be rewritten as

$$
\sum_{(\omega, \varepsilon)}^{*} \frac{1}{|\omega|} Z(\omega)
$$

where the sum $\sum^{*}$ is restricted in an obvious manner. Equivalently one can sum over equivalence classes $[(\omega, \varepsilon)]$ for the relation $\sim$, so that the above sum is

$$
=\sum_{[(\omega, \varepsilon)]}^{* *} \frac{\operatorname{card}[(\omega, \varepsilon)]}{|\omega|} Z(\omega) .
$$

The classes $[(\omega, \varepsilon)]$ appearing in the sum correspond to "extended orbits" of the form

$$
x, \bar{\psi}_{\omega_{1}} x, \ldots,\left(\psi_{\omega_{m}} \circ \cdots \circ \psi_{\omega_{1}}\right)^{-} x=x,
$$

where $\bar{\psi}$ denotes as usual the extension of $\psi$ by continuity to the closure of the interval of definition. Consider the values $k(i)$ (with $i=l, \ldots, n$ ) of $k$ such that $1 \leqq k \leqq m$ and $\left(\psi_{\omega_{k-1}} \circ \cdots \circ \psi_{\omega_{1}}\right)^{-} x$ is an endpoint $u_{\omega_{k}}$ or $v_{\omega_{k}}$ of $J_{\omega_{k}}$. We let $k(1)<k(2)<\cdots<k(n)$ and call $\omega^{(1)}, \ldots, \omega^{(n)}$ the pieces of $\omega$ such that $\omega^{(1)}=$ $\left(\omega_{l_{k(1)}}, \ldots, \omega_{l_{k(2)-1}}\right)$ etc. We have thus $Z(\omega)=Z\left(\omega^{(1)}\right) \cdots Z\left(\omega^{(n)}\right)$.

By construction, among the $n$ circular permutations of $\{1,2, \ldots, n\}$ generated by $1 \rightarrow 2 \rightarrow \cdots \rightarrow n \rightarrow 1$, there are $n(\omega, \varepsilon)=|\omega| / \operatorname{card}[(\omega, \varepsilon)]$ which leave

$$
\left(\xi_{1}, \omega^{(1)}\right),\left(\xi_{2}, \omega^{(2)}\right), \ldots,\left(\xi_{n}, \omega^{(n)}\right)
$$

fixed, hence the number of equivalence classes of permutations is $n / n(\omega, \varepsilon)$. The sum written above is thus

$$
\begin{aligned}
& =\sum_{[(\omega, \varepsilon)]}^{* *} \frac{n}{n(\omega, \varepsilon)} \cdot \frac{1}{n} Z\left(\omega^{(1)}\right) \cdots Z\left(\omega^{(n)}\right) \\
& =\sum_{n} \frac{1}{n} \sum_{\xi_{1} \cdots \xi_{n}} T_{\xi_{1} \xi_{2}} T_{\xi_{2} \xi_{3}} \cdots T_{\xi_{n-1} \xi_{n}} T_{\xi_{n} \xi_{1}}
\end{aligned}
$$

proving $(*)$. Therefore

$$
\zeta_{0}(Z)=\exp +\sum_{n} \frac{1}{n} \operatorname{tr} T^{n}=\exp \operatorname{tr}(-\log (1-T))=\operatorname{det}(1-T(Z))^{-1} .
$$

Given $\varepsilon>0$, let $\chi_{\eta}^{\varepsilon}$ be the characteristic function of $(|\eta|,|\eta|+\varepsilon)$ when $\operatorname{sign} \eta=+$, of $(|\eta|-\varepsilon,|\eta|)$ when sign $\eta=-$. Also write $x \rightarrow \xi$ when $\operatorname{sign} \xi \cdot(x-|\xi|) \downarrow 0$, and 
let $\sum_{\omega: \xi}$ be the sum over those $\omega$ such that $u_{\omega}+$ or $v_{\omega}-$ is $\xi$. Then one checks that

$$
\sum_{n \geqq 1}\left(T^{n}\right)_{\check{\xi} \eta}=\sum_{m \geqq 1} \lim _{\varepsilon \rightarrow 0} \lim _{x \rightarrow \varepsilon} \sum_{\omega \cdot \xi} z_{\omega}\left[\left(\mathscr{M}^{m-1} \chi_{n}^{\varepsilon}\right)\left(\psi_{\omega}(x)\right)\right] .
$$

Therefore $\operatorname{det}(1-T(Z))^{-1}$ is holomorphic when $R(Z)<1$. By symmetry, $\zeta_{0}(Z)$ is holomorphic when $\min (R(Z), \widehat{R}(Z))<1$, proving the lemma.

Write now $\chi_{|n|}(x)=\operatorname{del}(x-|n|)$ and

then we have

$$
\left(\mathscr{M}^{m-1}\right)_{ \pm}=\frac{1}{2}\left(\mathscr{M}_{Z}^{m-1} \pm \mathscr{M}_{\varepsilon Z}^{m-1}\right)
$$

$$
T_{\xi \eta}^{(m)}=\sum_{\omega: \xi} z_{\omega}\left[\left(\mathscr{M}^{m-1}\right)_{ \pm} \chi_{|\eta|}\right]\left(\bar{\psi}_{\omega}|\xi|\right)
$$

with the $\operatorname{sign} \pm=\varepsilon_{\omega} \operatorname{sign} \xi \cdot \operatorname{sign} \eta$. Therefore $T_{\xi \eta}$ is a holomorphic function of $Z$ when $\widehat{R}^{\#}(Z)<1$ and 1 is not an eigenvalue of $\mathscr{M}_{Z} \mid \mathscr{B}_{\infty}$ or $\mathscr{M}_{\varepsilon Z} \mid \mathscr{B}_{\infty}$. This justifies Remark 1.8 .

\section{Appendix A. Proof of Theorem A}

Let $\varepsilon_{\omega}= \pm 1$ for $\omega=1, \ldots, N$. Fixing $\left(J_{\omega}\right)$ and $\left(\varepsilon_{\omega}\right)$, let $P$ be the space of families $\psi=\left(\psi_{\omega}\right)$ such that each $\psi_{\omega}: J_{\omega} \rightarrow(-1,1)$ is continuous and strictly increasing if $\varepsilon_{\omega}=+1$, or strictly decreasing if $\varepsilon_{\omega}=-1$. We denote by $C^{r}\left(\bar{J}_{\omega}\right)$ the space of $C^{r}$ functions on the closure $\bar{J}_{\omega}$ of $J_{\omega}$, and write

$$
\begin{aligned}
P^{1}= & \left\{\psi:\left(\psi_{\omega}\right) \text { extends to }\left(\bar{\psi}_{\omega}\right) \in \bigoplus_{\omega} C^{1}\left(\bar{J}_{\omega}\right),\right. \\
& \text { and the derivatives } \left.\bar{\psi}_{\omega} \text { vanish on } \bar{J}_{\omega} \backslash J_{\omega}\right\}, \\
P^{\text {pol }}= & \left\{\psi \in P^{1}: \text { the } \psi_{\omega} \text { are polynomials }\right\} .
\end{aligned}
$$

We use the topology of $P, P^{1}$ induced by $\oplus C^{0}\left(\bar{J}_{\omega}\right), \oplus C^{1}\left(\bar{J}_{\omega}\right)$. In particular $P^{\text {pol }}$ is dense in $P, P^{1}$.

For finite $M$ we define

$$
\begin{aligned}
& F_{M}=\left\{\psi: \text { Fix } \psi_{\omega} \text { is finite when }|\omega| \leqq M\right\}, \\
& P_{M}=\left\{\psi: \bar{\psi}_{\omega}\left(u_{\omega}\right) \neq u_{\omega} \text { and } \bar{\psi}_{\omega}\left(v_{\omega}\right) \neq v_{\omega} \text { when }|\omega| \leqq M \text { and } J_{\omega} \neq \emptyset\right\} .
\end{aligned}
$$

Equivalently we may define $P_{M}$ as the set of those $\psi$ such that $\bar{\psi}_{\omega}\left(u_{\omega_{1}}\right)$ (if defined) is $\neq u_{\omega_{1}}$, and $\bar{\psi}_{\omega}\left(v_{\omega_{1}}\right)$ (if defined) is $\neq v_{\omega_{1}}$, when $|\omega| \leqq M$. We also write

$$
F_{\infty}=\bigcap_{M} F_{M}, \quad P_{\infty}=\bigcap_{M} P_{M} .
$$

Note that $P_{M}$ is open in $P$.

A.1. Lemma. If $\psi \in F_{M} \cap P_{M}$ and $|\omega| \leqq M$, we have

$$
L\left(\psi_{\omega}\right)=\sum_{x \in \mathrm{Fix} \psi \omega} L\left(x, \psi_{\omega}\right) .
$$

This follows from part (c) of the lemma of Sect.1.1. 
Let $|\omega|$ be the class of $\omega$ under circular permutations, and say that $[\omega]$ is prime if $\omega$ is not the periodic repetition of $n$ copies of a sequence $\omega^{\prime}$ with $\left|\omega^{\prime}\right|<|\omega|$. Then we have the product formula

where

$$
\zeta(Z)=\prod_{[\omega] \text { prime }} G_{[\omega]}(Z)
$$

$$
G_{[\omega]}(Z)=\exp \sum_{n=1}^{\infty} \frac{1}{n} L\left(\psi_{\omega}^{n}\right) Z(\omega)^{n} .
$$

The following possibilities exist

(0) $\varepsilon(\omega)= \pm 1, L\left(\psi_{\omega}\right)=0$, then $G_{[\omega]}(Z)=1$,

(1) $\varepsilon(\omega)=+1, L\left(\psi_{\omega}\right)=-1$, then $G_{[\omega]}(Z)=1-Z(\omega)$,

(2) $\varepsilon(\omega)=+1, L\left(\psi_{\omega}\right)=1$, then $G_{[\omega]}(Z)=(1-Z(\omega))^{-1}$,

(3) $\varepsilon(\omega)=-1, \quad L\left(\psi_{\omega} \circ \psi_{\omega}\right)=1$, then $G_{[\omega]}(Z)=(1-Z(\omega))^{-1}$,

(4) $\varepsilon(\omega)=-1, \quad L\left(\psi_{\omega} \circ \psi_{\omega}\right)=-1$, then $G_{[\omega]}(Z)=1+Z(\omega)$.

A.2. Lemma. $\zeta(Z)$ and $1 / \zeta(Z) \in \mathbb{Z} \llbracket z_{1}, \ldots, z_{N} \rrbracket$. If $\mathfrak{I}_{M+1}$ is the ideal of elements of order $\geqq M+1$ in $\mathbb{Q} \llbracket z_{1}, \ldots, z_{N} \rrbracket$, then $\zeta(Z)\left(\bmod \mathfrak{I}_{M+1}\right)$ is locally constant on $P_{M}$.

This follows from the product formula given above and the definition of $P_{M} . \square$

A.3. Lemma. If $\psi$ satisfies $\psi_{\omega} J_{\omega}>a_{L}$ for $\omega=1, \ldots, N$, we have

$$
\zeta=D=1 \text {. }
$$

Clearly $\psi \in F_{\infty} \cap P_{\infty}$. In fact Fix $\psi_{\omega}=\emptyset$ for all $\omega$, hence $\zeta(Z)=1$.

In the present situation $\mathscr{M}^{m}=0$ for $m>1$. We have thus

$$
\begin{aligned}
D_{i j} & =\delta_{i j}+A_{i}, \\
A_{l} & =\frac{1}{2}\left[\sum_{\omega: u_{\omega}=a_{l}} z_{\omega}-\sum_{\omega: v_{\omega}=a_{i}} z_{\omega}\right],
\end{aligned}
$$

i.e., the kneading matrix $\left[D_{l j}\right]$ is the sum of the unit matrix $\left[\delta_{i j}\right]$ and a matrix of rank $\leqq 1$. Therefore

$$
D=\operatorname{det}\left[D_{i j}\right]=1+\sum_{i} A_{i}=1+\frac{1}{2}\left(\sum_{\omega} z_{\omega}-\sum_{\omega} z_{\omega}\right)=1,
$$

which concludes the proof.

A.4. Lemma. Let $\tilde{J}_{\omega}, \tilde{\zeta}, \tilde{D}$ correspond to $J_{\omega}, \zeta, D$ when $\tilde{\psi}$ replaces $\psi$. Given $M \geqq 1$, we assume that $\tilde{\psi}$ is sufficiently close to $\psi$ in $P$ (in particular $\left.\tilde{J}_{\omega}=J_{\omega}\right)$, and that

$$
\begin{gathered}
\tilde{J}_{\omega} \supset J_{\omega}, \quad \tilde{\psi}_{\omega} \tilde{J}_{\omega} \subset \psi_{\omega} J_{\omega}, \\
J_{\omega} \cap \psi_{\omega} J_{\omega}=\emptyset \Rightarrow \tilde{J}_{\omega} \cap \tilde{\psi}_{\omega} \tilde{J}_{\omega}=\emptyset
\end{gathered}
$$

for $|\omega| \leqq M$. Then

$$
\begin{gathered}
\tilde{\zeta}(Z)=\zeta(Z) \quad\left(\bmod \mathfrak{I}_{M+1}\right), \\
\tilde{D}(Z)=D(Z) \quad\left(\bmod \mathfrak{I}_{M+1}\right) .
\end{gathered}
$$


Furthermore, if

$$
\overline{\tilde{\psi}}_{\omega}\left(u_{\omega}\right) \neq \tilde{\psi}_{\omega}\left(u_{\omega}\right), \quad \overline{\tilde{\psi}}_{\omega}\left(v_{\omega}\right) \neq \tilde{\psi}_{\omega}\left(v_{\omega}\right)
$$

for $|\omega| \leqq M$, we may assume that

$$
\overline{\tilde{\psi}}_{\omega}\left(u_{\omega}\right), \overline{\tilde{\psi}}_{\omega}\left(v_{\omega}\right) \notin\left\{a_{1}, \ldots, a_{L}\right\}
$$

when $|\omega| \leqq M$ (in particular $\tilde{\psi} \in P_{M}$ ).

First note that if $J_{\omega}=\emptyset$, then $\tilde{J}_{\omega}=\emptyset$ (because (1) gives $\tilde{\psi}_{\omega} \tilde{J}_{\omega} \subset \psi_{\omega} J_{\omega}=\emptyset$ ). If $J_{\omega} \neq \emptyset$, the set $\tilde{J}_{\omega}$ is close to $J_{\omega}$ and the set $\tilde{\psi}_{\omega} \tilde{J}_{\omega}$ is close to $\psi_{\omega} J_{\omega}$; then (2) and the inclusions (1) imply that $L\left(\tilde{\psi}_{\omega}\right)=L\left(\psi_{\omega}\right)$ for $|\omega| \leqq M$ (the argument is the same as for part (a) of the lemma in Sect. 1.1: check the list of cases when $L\left(\psi_{\omega}\right)=1,-1$, or 0$)$. This implies $\tilde{\zeta}(Z)=\zeta(Z)\left(\bmod \mathfrak{I}_{M+1}\right)$.

Suppose that $u_{\omega}=u_{\omega_{1}}=a_{l}$. When $\tilde{\psi} \rightarrow \psi$, then

$$
\bar{\psi}_{\omega}\left(a_{i}\right) \rightarrow \bar{\psi}_{\omega}\left(a_{i}\right)
$$

and the inclusion (1) implies that the above limit is reached on the same side as the limit

$$
\psi_{\omega}(x) \rightarrow \bar{\psi}_{\omega}\left(a_{i}\right)
$$

when $x \downarrow a_{i}$. Therefore (for $\tilde{\psi}$ close to $\psi$ )

and similarly

$$
\tilde{D}_{i j}^{(m)+}=D_{i j}^{(m)+}
$$

This means that

$$
\tilde{D}_{l j}^{(m)-}=D_{l j}^{(m)-}
$$

$$
\tilde{D}_{l j}=D_{\imath j}\left(\bmod \mathfrak{I}_{M+1}\right)
$$

hence

$$
\tilde{D}(Z)=D(Z)\left(\bmod \mathfrak{I}_{M+1}\right) .
$$

The last statement of the lemma follows from the fact that the numbers

$$
\left|\overline{\tilde{\psi}}_{\omega}\left(u_{\omega}\right)-\bar{\psi}_{\omega}\left(u_{\omega}\right)\right|, \quad\left|\overline{\tilde{\psi}}_{\omega}\left(v_{\omega}\right)-\bar{\psi}_{\omega}\left(v_{\omega}\right)\right|
$$

are in an arbitrarily small interval $(0, \delta)$.

A.5. Proof of the Theorem. It will suffice to prove Theorem A $\left(\bmod \mathfrak{I}_{M+1}\right)$ for all integers $M \geqq 1$. We fix $M$ for the rest of the argument.

For small $\delta>0$, let the homeomorphism $\varphi_{\omega}:\left(u_{\omega}, v_{\omega}\right) \rightarrow\left(u_{\omega}+\delta, v_{\omega}-\delta\right)$ be the identity on $\left[u_{\omega}+2 \delta, v_{\omega}-2 \delta\right]$ and a contraction on $\left(u_{\omega}, u_{\omega}+2 \delta\right)$ and $\left(v_{\omega}-\right.$ $\left.2 \delta, v_{\omega}\right)$. We define $\widetilde{\psi}_{\omega}=\psi_{\omega} \circ \varphi_{\omega}$ for $\omega=1, \ldots, N$. Writing

$$
\boldsymbol{\omega}=\left(\omega_{1}, \ldots, \omega_{m}\right), \quad \boldsymbol{\omega}^{\prime}=\left(\omega_{1}, \ldots, \omega_{m-1}\right),
$$

we may assume that the length of $J_{\omega_{m}} \cap \psi_{\omega^{\prime}} J_{\omega^{\prime}}$ is $\geqq a>0$ whenever $|\omega| \leqq 2 M$ and $J_{\omega} \neq \emptyset$. If $\delta$ is sufficiently small we may also assume that the length of $J_{\omega_{m}} \cap$ $\psi_{\omega^{\prime}} J_{\omega^{\prime}}$ is $\geqq a>0$ whenever $|\omega| \leqq 2 M$ and $J_{\omega} \neq \emptyset$. If $\delta$ is sufficiently small we may also assume that the length of $J_{\omega_{m}} \cap \widetilde{\psi}_{\omega^{\prime}} \tilde{J}_{\omega^{\prime}}$ is $\geqq b=\frac{a}{2}$. Note that

$$
\widetilde{\psi}_{\omega} \tilde{J}_{\omega}=\widetilde{\psi}_{\omega_{m}}\left(J_{\omega_{m}} \cap \widetilde{\psi}_{\omega^{\prime}} \tilde{J}_{\omega^{\prime}}\right)
$$


Assuming now that $2 \delta<b$, we see by induction on $|\omega|$ that

$$
\widetilde{\psi}_{\omega} \tilde{J}_{\omega} \subset \psi_{\omega} J_{\omega}
$$

Writing $\omega(k)=\left(\omega_{1}, \ldots, \omega_{k}\right)$ we also see (by induction on $k$, and assuming $\delta$ small enough) that

$$
\widetilde{\psi}_{\omega(k)} J_{\omega} \subset \psi_{\omega(k)} J_{\omega} .
$$

In particular $\widetilde{\psi}_{\omega}$ is defined on $J_{\omega}$, i.e.,

$$
\tilde{J}_{\omega} \supset J_{\omega}
$$

The condition (1) of Lemma A.4 is thus satisfied when $|\omega| \leqq 2 M$. Writing $\left(\omega_{1}, \ldots, \omega_{m}, \omega_{1}, \ldots, \omega_{m}\right)=2 \omega$, we have

$$
J_{\omega} \cap \psi_{\omega} J_{\omega}=\psi_{\omega} J_{2 \omega} .
$$

Therefore (2) for $|\omega| \leqq M$ follows from the implication $\psi_{2 \omega} J_{2 \omega}=\emptyset \Rightarrow$ $\widetilde{\psi}_{2 \omega} \tilde{J}_{2 \omega}=\emptyset$ (which follows from (1)). By induction on $m$ we see that (3) also holds.

We may now approximate $\widetilde{\psi}$ in $P^{0}$ by $\psi^{1} \in P^{\text {pol }}$ while respecting the conditions (1), (2), and (3). Lemma A.4 thus shows that, to prove Theorem A, it suffices to prove that

$$
\zeta^{1}(Z)=D^{1}(Z)\left(\bmod \Im_{M+1}\right)
$$

where $\zeta^{1}(Z)$ and $D^{1}(Z)$ are constructed with $\psi^{1} \in P^{\text {pol }}$ such that

$$
\tilde{\psi}_{\omega}^{1}\left(u_{\omega}\right), \tilde{\psi}_{\omega}^{1}\left(v_{\omega}\right) \notin\left\{a_{1}, \ldots, a_{L}\right\}
$$

for $|\omega| \leqq M$.

Let $\psi^{0} \in P^{\text {pol }}$ be defined as in Lemma A.3, and $\psi^{\lambda}=(1-\lambda) \psi^{0}+\lambda \psi^{1}$. By definition, $\psi^{\lambda}=\left(\psi_{1}^{\lambda}, \ldots, \psi_{N}^{\lambda}\right)$ is an $N$-tuple of polynomials, none of which is affine $\left[\bar{\psi}_{\omega}^{\lambda}\right.$ is non-constant, with derivatives vanishing at $\left.u_{\omega}, v_{\omega}\right]$; in particular $\psi^{\lambda} \in F_{\infty}$. Note that the functions $(x, \lambda) \mapsto \psi_{\omega}^{\lambda}(x), \psi_{\omega}^{\lambda}$ are polynomials, and extend therefore naturally to $\mathbb{R}^{2}$. Until further notice we shall use these extended definitions. The polynomials $\lambda \mapsto \psi_{\omega}^{\hat{\lambda}}\left(a_{i}\right)-a_{j}$ (defined for all $\omega=\left(\omega_{1}, \ldots, \omega_{m}\right)$ with $1 \leqq m \leqq M$ and $i, j \in\{1, \ldots, L\})$ may be assumed not to vanish at $\lambda=0,1$. Therefore there is a finite set $\Lambda \subset(0,1)$ of values of $\lambda$ such that

$$
\psi_{\omega}^{\lambda}\left(a_{i}\right)=a_{j}
$$

for some $i, j$, and $\omega$. If $\zeta^{\lambda}$ and $D^{\lambda}$ denote $\zeta$ and $D$ computed with $\psi^{\lambda}$, we see that $\zeta^{\lambda}\left(\bmod \mathfrak{I}_{M+1}\right)$ remains constant in each interval of $[0,1] \backslash \Lambda$ [see Lemma A.2] and the same is true for $D^{\lambda}\left(\bmod \Im_{M+1}\right)$ [because the $D_{i j}^{(m) \pm}$ are constant].

In view of Lemma A.3, in order to prove Theorem A it suffices to show that $\zeta^{\lambda}$ and $D^{\lambda}$ are multiplied by the same factor $\left(\bmod \mathfrak{J}_{M+1}\right)$ whenever $\lambda$ crosses a point of $\Lambda$.

The changes of sign of the $\psi_{\omega}^{\lambda}\left(a_{l}\right)-a_{j}$ when $\lambda$ crosses an element of $\Lambda$ may be complicated. We shall make them simpler by modifying $\left(\psi^{i}\right)$ to obtain a family $\left(\widetilde{\psi}^{\lambda}\right)$ with nonlinear dependence on $\lambda$.

Let us assume that $(x, \lambda) \mapsto \widetilde{\psi}_{\omega}^{\lambda}(x)$, defined on $\mathbb{R}^{2}$, is $C^{\infty}$ close to $(x, \lambda) \mapsto \psi_{\omega}^{\lambda}(x)$, for $\omega=1, \ldots, N$, and construct $\widetilde{\psi}_{\omega}^{\lambda}=\widetilde{\psi}_{\omega_{m}}^{\lambda} \circ \cdots \circ \widetilde{\psi}_{\omega_{1}}^{\lambda}$. In particular the functions 
$\lambda \mapsto \widetilde{\psi}_{\omega}^{\lambda}\left(a_{i}\right)-a_{j}$ are $C^{\infty}$ close to the polynomials $\lambda \mapsto \psi_{\omega}^{\lambda}\left(a_{l}\right)-a_{j}$ and may be assumed not to vanish at $\lambda=0,1$. Let $\widetilde{\Lambda}$ be the set of all $\lambda \in(0,1)$ for which $\widetilde{\psi}_{\omega}^{\lambda}\left(a_{i}\right)=a_{j}$ for some $i, j$, and some $\omega$ with $|\omega| \leqq M$. Then card $\widetilde{\Lambda}$ is bounded by the sum (over $i, j, \omega)$ of the degrees of the polynomials $\lambda \mapsto \psi_{\omega}^{\lambda}\left(a_{l}\right)-a_{j}$, hence uniformly in $\left(\widetilde{\psi}^{\lambda}\right)$ for $\left(\widetilde{\psi}^{\lambda}\right)$ in a suitable $C^{\infty}$ neighborhood of $\left(\psi^{\lambda}\right)$. We shall use the uniformity of this bound in a moment.

Given $\lambda_{0} \in \Lambda$ we construct an oriented graph $\Gamma$ as follows. The set of vertices of $\Gamma$ is

$$
X=\left\{\xi \in \mathbb{R}: \text { there exist } \omega=\left(\omega_{1}, \ldots, \omega_{m}\right) \text { with } 1 \leqq m \leqq M, i, j \in\{1, \ldots, L\}\right.
$$

and $k \in\{0, \ldots, m\}$ such that $\left.\psi_{\omega_{k}}^{\lambda_{0}} \circ \cdots \circ \psi_{\omega_{1}}^{\lambda_{0}} a_{i}=\xi, \psi_{\omega_{m}}^{\lambda_{0}} \circ \cdots \circ \psi_{\omega_{k+1}}^{\lambda_{0}} \xi=a_{j}\right\}$.

The set of arrows is

$$
\left\{(\xi, \omega): 1 \leqq \omega \leqq N, \xi \in X \text { and } \psi_{\omega}^{\lambda_{0}} \xi \in X\right\}
$$

The arrow $(\xi, \omega)$ starts at $\xi$ and goes to $\eta=\psi_{\omega}^{\lambda_{0}} \xi$; there may thus be several arrows $\xi \Rightarrow \eta$. An arrow $(\xi, \omega): \xi \Rightarrow \eta$ may be removed from the graph corresponding to $\lambda_{0}$ by a $C^{\infty}$ small change of $(x, y) \mapsto \psi_{(i)}^{\lambda}(x)$ near $\left(\xi, \lambda_{0}\right)$. Repeating this operation, we can arrange that $\left(\psi^{\lambda}\right)$ is replaced by $\left(\widetilde{\psi}^{\lambda}\right)$ such that the graph corresponding to $\lambda_{0}$ consists of a simple arc $a_{l} \Rightarrow \xi \Rightarrow \eta \Rightarrow \cdots \Rightarrow a_{j}$ (where $a_{j}$ may be equal to $\left.a_{i}\right)$ and $\xi, \eta, \ldots \notin\left\{a_{i}, \ldots, a_{L}\right\}$. This means that there are unique $i, j$, and $\omega^{*}$ with $\left|\omega^{*}\right| \leqq M$ such that $\widetilde{\psi}_{\omega^{*}}^{\lambda_{0}}\left(a_{i}\right)=a_{J}$ and $\tilde{\psi}_{\omega_{k}^{*}}^{\lambda_{0}} \circ \cdots \circ \widetilde{\psi}_{\omega_{1}^{*}}^{\lambda_{0}} a_{l} \notin\left\{a_{1}, \ldots, a_{L}\right\}$ for $k<\left|\omega^{*}\right|$. By a small change of $\left(\widetilde{\psi}^{\lambda}\right)$ near $\lambda=\lambda_{0}$ we may further achieve that Fix $\widetilde{\psi}_{\omega}^{\lambda_{0}}$ is finite when $|\omega| \leqq M$, and that the fixed points are not degenerate (i.e., the derivative of $\widetilde{\psi}_{\omega}^{\hat{\lambda}_{0}}$ at $\xi \in$ Fix $\widetilde{\psi}_{\omega}^{\hat{\lambda}_{0}}$ is $\left.\neq 1\right)$. Note that the families $\left(\psi^{\lambda}\right)$ and $\left(\widetilde{\psi}^{\lambda}\right)$ coincide outside of a small neighborhood of $\lambda_{0}$; to obtain $\tilde{\Lambda}$ from $\Lambda$ we have replaced $\lambda_{0}$ by a finite set $\left\{\lambda_{0}, \lambda_{0}^{\prime}, \ldots\right\}$.

We may now start again the above process with a new element $\tilde{\lambda}_{0}$ of $\widetilde{\Lambda}$ (being careful to leave $\left(\widetilde{\psi}^{\hat{\lambda}_{0}}\right)$ unchanged). Since the cardinality of the sets $\Lambda, \widetilde{\Lambda}, \ldots$ is uniformly bounded, after a finite number of steps the family $\left(\psi^{\lambda}\right)$ is replaced by $\left(\Psi^{\lambda}\right)$ with the following properties.

(a) $\Psi^{\lambda} \in P^{1},(x, \lambda) \mapsto \Psi^{\lambda}(x)$ is $C^{\infty}$, and $\Psi^{0}=\psi^{0}, \Psi^{1}=\psi^{1}$.

(b) For $\lambda$ outside of a finite set $\Lambda^{*}$,

$$
\Psi_{\omega}^{\lambda}\left(a_{l}\right) \neq a_{J}
$$

if $i, j \in\{1, \ldots, L\}$ and $|\omega| \leqq M$.

(c) If $\lambda \in \Lambda^{*}$ there are unique $i, j \in\{1, \ldots, L\}$ and $\omega^{*}$ with $\left|\omega^{*}\right| \leqq M$ such that

$$
\Psi_{\omega^{*}}^{\lambda}\left(a_{i}\right)=a_{j}
$$

and $\Psi_{\omega_{k}^{*}}^{\lambda} \circ \cdots \circ \Psi_{\omega_{1}^{*}}^{\lambda} a_{i} \notin\left\{a_{1}, \ldots, a_{L}\right\}$ if $k<\left|\omega^{*}\right|$.

(d) If $\lambda \in \Lambda^{*}$, and $|\omega| \leqq M$, then Fix $\Psi_{\omega}^{\lambda}$ is finite and the fixed points $\xi \in \operatorname{Fix} \Psi_{\omega}^{\lambda}$ are nondegenerate, i.e., $\left(\Psi_{\omega}^{\lambda}\right)^{\prime}(\xi) \neq 1$. 
To prove the theorem it suffices therefore to check (under the conditions (a), (b), (c), (d)) that the zeta function $\zeta$ and the kneading determinant $D$ associated with $\left(\Psi^{\lambda}\right)$ are multiplied by the same factor $\left(\bmod \mathfrak{I}_{M+1}\right)$ when $\lambda$ crosses a point of $\Lambda^{*}$. This is done in the following lemma.

We return now to the standard notation where $\psi_{\omega}$ is defined only on $J_{\omega}$ and $\bar{\psi}_{\omega}$ is the extension of $\psi_{\omega}$ by continuity to the closure $\bar{J}_{\omega}$; similarly for $\psi_{\omega}, \bar{\psi}_{\omega}$.

A.6. Lemma. Let $\psi \in P^{1}$ be such that there are unique $i, j \in\{1, \ldots, L\}$ and $\omega^{*}$ with $\left|\omega^{*}\right| \leqq M$ such that

$$
\bar{\psi}_{\omega^{*}}\left(a_{i}\right)=a_{j}
$$

and $\bar{\psi}_{\omega_{k}^{*}} \circ \cdots \circ \bar{\psi}_{\omega_{1}^{*}} a_{l} \notin\left\{a_{1}, \ldots, a_{L}\right\}$ if $k<\left|\omega^{*}\right|$. We further assume that whenever $|\omega| \leqq M$ the set Fix $\psi_{\omega}$ is finite and consists of nondegenerate fixed points $\xi$, i.e., $\psi_{\omega}^{\prime}(\bar{\xi}) \neq 1$.

Then if $\psi^{>}, \psi^{<}$are sufficiently close to $\psi$ in $P^{1}$ and such that $\psi_{\omega^{*}}^{>}\left(a_{1}\right)>a_{j}$, $\psi_{\omega^{*}}^{<}\left(a_{i}\right)<a_{j}$ we have

$$
\zeta^{>} / \zeta^{<}=D^{>} / D^{<}\left(\bmod \mathfrak{I}_{M+1}\right)
$$

where $\zeta^{\gtrless}, D^{\gtrless}$ denote $\zeta, D$ computed from $\psi^{\gtrless}$

We first observe that $\zeta^{>} / \zeta^{<}=D^{>} / D^{<}=1\left(\bmod \mathfrak{I}_{M+1}\right)$ unless $a_{i}$ is one of the endpoints $u_{\omega_{1}^{*}}$ or $v_{\omega_{1}^{*}}$ of $J_{\omega_{1}^{*}}$. Using the symmetry $x \rightarrow-x$ of $\mathbb{R}$ we see that it suffices to consider the situation where $u_{\omega_{1}^{*}}=a_{i}$. In this case we claim that we have $\left(\bmod \mathfrak{I}_{M+1}\right)$

$$
\begin{array}{lll}
\zeta^{>}=\zeta^{<}, & D^{>}=D^{<} & \text {if } j \neq i, \\
\zeta^{>}=\zeta^{<} \cdot\left(1-Z\left(\omega^{*}\right)\right)^{-1}, & D^{>}=D^{<} \cdot\left(1-Z\left(\omega^{*}\right)\right)^{-1} & \text { if } j=i .
\end{array}
$$

We first discuss the easy proof of the formulas for the zeta function. If $j \neq i$, then $\zeta\left(\bmod \mathfrak{I}_{M+1}\right)$ is locally constant at $\psi($ Lemma A.2), hence $\zeta>=\zeta<$.

Let $j=i$. We have $\bar{\psi}_{\omega^{*}} a_{i}=a_{i}$. The point $a_{i}$ bifurcates into an attracting fixed point for $\psi_{\omega^{*}}^{>}$, absent for $\psi_{\omega^{*}}^{<}$(see the figure). Apart from the periodic orbit thus created, the periodic orbits for $\psi, \psi^{>}, \psi^{<}$correspond to each other, with the same weight, up to order $\geqq M+1$, if $\psi^{>}$and $\psi^{<}$are sufficiently close to $\psi$ in $P^{1}$. Therefore

$$
\zeta^{>}=\zeta^{<}\left(1-Z\left(\omega^{*}\right)\right)^{-1}
$$

as announced.

Graph of $\psi_{\omega}$. The graph of $\psi_{\omega^{*}}^{>}$(resp. $\psi_{\omega^{*}}^{<}$) is obtained by pushing the graph of $\psi_{\omega^{*}}$ upwards (resp. downwards).

We consider now the changes for $D$. Let $\delta D$ denote the jump of $D$ from $\psi^{<}$ to $\psi^{>}$and similarly for $\delta D_{i k}, \ldots$ We have $\delta D_{l k}^{(m)-}=0$, hence

$$
\begin{aligned}
\delta D_{i k} & =\frac{1}{2} \sum_{m=1}^{\infty} \delta D_{i k}^{(m)+}=\frac{1}{2} \sum_{m \geqq 1} \lim _{x \downarrow a_{l}}\left[\left(\left(\mathscr{M}^{>}\right)^{m} \alpha_{k}\right)(x)-\left(\left(\mathscr{M}^{<}\right)^{m} \alpha_{k}\right)(x)\right] \\
& =\frac{1}{2} Z\left(\omega^{*}\right) \sum_{n \geqq 0} \lim _{x \downarrow a_{i}}\left[\left(\left(\mathscr{M}^{>}\right)^{n} \alpha_{k}\right)\left(\psi_{\omega^{*}}^{>} x\right)-\left(\left(\mathscr{M}^{<}\right)^{n} \alpha_{k}\right)\left(\psi_{\omega^{*}}^{<} x\right)\right]
\end{aligned}
$$




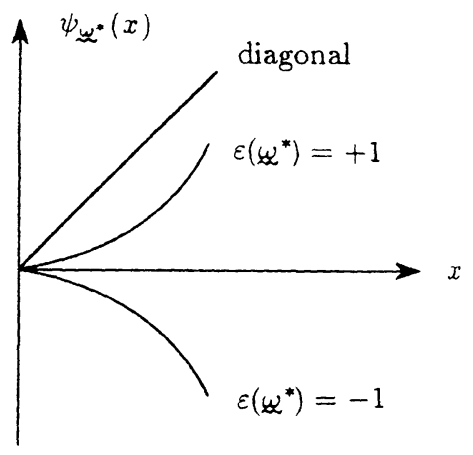

Fig. 1.

with obvious notation. Let $\Phi$ denote a function which is locally constant on $\mathbb{R}$ outside of $\left\{a_{1}, \ldots, a_{L}\right\}$, like $\chi_{\omega}$ or $\alpha_{k}$. If $\left|\omega^{*}\right|+|\omega| \leqq M$ we have

$$
\begin{aligned}
& \lim _{x \downarrow a_{l}}\left(\Phi \circ \psi_{\omega}^{>} \circ \psi_{\omega^{*}}^{>}\right)(x)=\lim _{x \downarrow a_{j}}\left(\Phi \circ \psi_{\omega}^{>}\right)(x), \\
& \lim _{x \downarrow a_{l}}\left(\Phi \circ \psi_{\omega}^{<} \circ \psi_{\omega^{*}}^{<}\right)(x)=\lim _{x \uparrow a_{j}}\left(\Phi \circ \psi_{\omega}^{<}\right)(x)=\lim _{x \uparrow a_{j}}\left(\Phi \circ \psi_{\omega}^{>}\right)(x),
\end{aligned}
$$

when $\psi^{<}$and $\psi^{>}$are sufficiently close to $\psi$ in $P^{1}$. Therefore $\left(\bmod \mathfrak{I}_{M+1}\right)$

$$
\delta D_{i k}=Z\left(\omega^{*}\right) \sum_{n \geqq 0} \frac{1}{2}\left[\lim _{x \downarrow a_{j}}\left(\left(M^{>}\right)^{n} \alpha_{k}\right)(x)-\lim _{x \uparrow a_{j}}\left(\left(M^{>}\right)^{n} \alpha_{k}\right)(x)\right]=Z\left(\omega^{*}\right) D_{j k}^{>} .
$$

If $i \neq j$, we have $D_{j k}^{>}=D_{j k}$. Therefore in $\delta\left[D_{i k}\right]$ the $i^{\text {th }}$ and $j^{\text {th }}$ line are proportional, giving $\delta D=0$, i.e., $D^{<}=D^{>}$.

If $i=j$, we have

$$
D^{>}-D^{<}=\delta D=Z\left(\omega^{*}\right) D^{>}
$$

hence

$$
D^{>}=D^{<} \cdot\left(1-Z\left(\omega^{*}\right)\right)^{-1}
$$

as announced.

\section{Appendix B. Generalized Transfer Operators}

As before, $\mathscr{B}$ denotes the Banach space of functions $\Phi: \mathbb{R} \rightarrow \mathbb{C}$ of bounded variation. We use on $\mathscr{B}$ the norm Var defined by

$$
\operatorname{Var} \Phi=\lim \left[\left|\Phi\left(a_{0}\right)\right|+\sum_{i=1}^{n}\left|\Phi\left(a_{l}\right)-\Phi\left(a_{i-1}\right)\right|+\left|\Phi\left(a_{n}\right)\right|\right],
$$

where the limit is taken over finite sets $\left\{a_{0}, \ldots, a_{n}\right\}$ (with $a_{0}<a_{1}<\cdots<a_{n}$ ) ordered by inclusion.

We also write

$$
\mathscr{B}_{\infty}=\{\Phi \in \mathscr{B}:\{x: \Phi(x) \neq 0\} \text { is countable }\}
$$


and let $\|\cdot\|^{\#}$ denote the quotient norm on $\mathscr{B}^{\#}=\mathscr{B} / \mathscr{B}_{\infty}$. We have then

$$
\|[\Phi]\|^{\#}=\operatorname{Var}^{\#} \Phi,
$$

where $\operatorname{Var}^{\#}$ is defined like Var, but with $\left\{a_{0}, \ldots, a_{n}\right\}$ ranging over the finite subsets of a generic dense set $\mathbf{R}$. By this we mean that the closure of $\mathbf{R}$ is $\mathbb{R}$, and that $\mathbf{R}$ is disjoint from any countable set given in advance. (For the definition of $\operatorname{Var}^{\#} \Phi$, the set to avoid is that of discontinuities of $\Phi$.) Using $\operatorname{Var}^{\#}$ it is easy to implement Remark 1.7, and obtain a \#-version of Theorem B.1 below.

We let $\Omega$ be a countable set and for each $\omega \in \Omega$ we suppose that

$\Lambda_{()}$is an interval of $\mathbb{R}$ (not necessarily open or closed).

$\psi_{\omega}: \Lambda_{\omega} \rightarrow \mathbb{R}$ is continuous and strictly monotone (i.e. $\psi_{\omega}: \Lambda_{\omega} \rightarrow \psi_{\omega} \Lambda_{\omega}$ is a homeomorphism).

$\varphi_{\omega}: \Lambda_{\omega} \rightarrow \mathbb{C}$ has bounded variation. We also assume that

$$
V=\sum_{\omega \in \Omega} \operatorname{Var} \varphi_{\omega}<\infty
$$

[In order to define $\operatorname{Var} \varphi_{\omega}$, we extend $\varphi_{\omega}$ to be 0 on $\mathbb{R} \backslash \Lambda_{\omega}$.]

We write $\varepsilon_{\omega}=+1$ if $\psi_{\omega}$ is increasing, -1 if $\psi_{\omega}$ is decreasing [we make an arbitrary choice if $\Lambda_{\omega}$ is reduced to one point or empty].

On the Banach space $\mathscr{B}$ we define the operators $\mathscr{M}$ and $\widehat{\mathscr{M}}$ such that

$$
\begin{aligned}
& \mathscr{M} \Phi(x)=\sum_{\omega} \varphi_{\omega}(x) \Phi\left(\psi_{\omega} x\right), \\
& \widehat{\mathscr{M}} \Phi(x)=\sum_{\omega} \varepsilon_{\omega} \varphi_{\omega}\left(\psi_{\omega}^{-1} x\right) \Phi\left(\psi_{\omega}^{-1} x\right) .
\end{aligned}
$$

[We let $\varphi_{\omega}(x) \Phi\left(\psi_{\omega} x\right)=0$ if $x \notin \Lambda_{\omega}$ and $\varphi_{\omega}\left(\psi_{\omega}^{-1} x\right) \Phi\left(\psi_{\omega}^{-1} x\right)=0$ if $x \notin \psi_{\omega} \Lambda_{\omega}$.] The operators $\mathscr{M}$ and $\widehat{\mathscr{M}}$ are bounded. If we denote by $\|M\|$ the norm of the operator $M$ acting on $\mathscr{B}$ (with the Var norm) and by $\|M\|_{0}$ the norm of the operator $M$ acting on bounded function (with the uniform norm $\|\cdot\|_{0}$ ) we have

$$
\|\mathscr{M}\|,\|\widehat{\mathscr{M}}\|,\|\mathscr{M}\|_{0},\|\widehat{\mathscr{M}}\|_{0}<V .
$$

We write

$$
\begin{aligned}
& R=\lim _{m \rightarrow \infty}\left(\left\|\mathscr{M}^{m}\right\|_{0}\right)^{1 / m}, \\
& \widehat{R}=\lim _{m \rightarrow \infty}\left(\left\|\widehat{\mathscr{M}^{m}}\right\|_{0}\right)^{1 / m} .
\end{aligned}
$$

The submultiplicativity of $m \mapsto\left\|\mathscr{M}^{m}\right\|_{0},\left\|\widehat{\mathscr{M}}^{m}\right\|_{0}$ guarantees the existence of the limits; $R$ and $\widehat{R}$ are in fact the spectral radii of $\mathscr{M}, \widehat{\mathscr{M}}$ acting on bounded functions $X \rightarrow \mathbb{C}$. In general $R \neq \widehat{R}$.

B.1. Theorem. ${ }^{1}$ (a) The spectral radius of $\mathscr{M}$ acting on $\mathscr{B}$ is $\leqq \max (R, \widehat{R})$ and $\geqq \widehat{R}$.

(b) The essential spectral radius of $\mathscr{M}$ is $\leqq \widehat{R}$.

(c) If $\varphi_{\omega} \geqq 0$ for all $\omega$, the spectral radius of $\mathscr{M}$ is $\geqq R$. If furthermore $\widehat{R}<R$, then $R$ is an eigenvalue of $\mathscr{M}$, and there is a corresponding eigenfunction $\Phi_{R} \geqq 0$.

1 This is an improved version of the theorem of [4]. 
Note that $\mathscr{M}, \widehat{\mathscr{M}}$ play symmetric roles: $\mathscr{M}$ may be replaced by $\widehat{\mathscr{M}}$ in the theorem if $R, \widehat{R}$ are interchanged.

It will be convenient to assume that all $\Lambda_{\omega}$ and $\psi_{\omega} \Lambda_{\omega}$ are contained in $(-1,+1)$. This can be achieved by the embedding $\mathbb{R} \rightarrow(-1,+1)$ given by $x \rightarrow$ $x\left(1+x^{2}\right)^{-1 / 2}$. We can then also extend the $\psi_{\omega}$ to homeomorphisms $\mathbb{R} \rightarrow \mathbb{R}$, and take $\varphi_{\omega} \mid\left(\mathbb{R} \backslash \Lambda_{\omega}\right)=0$.

The proof of the theorem will use bilinear forms on $\mathscr{B}$ which we now introduce. If $\Phi, \Psi: \mathbb{R} \rightarrow \mathbb{C}$ are of bounded variation we may define

$$
\begin{aligned}
\langle\Psi, \Phi\rangle_{+} & =\lim \sum_{l=1}^{n} \Psi\left(a_{l}\right)\left(\Phi\left(a_{l}\right)-\Phi\left(a_{i-1}\right)\right) \\
\langle\Psi, \Phi\rangle_{-} & =\lim \sum_{l=1}^{n} \Psi\left(a_{l-1}\right)\left(\Phi\left(a_{l}\right)-\Phi\left(a_{l-1}\right)\right), \\
\langle\Psi, \Phi\rangle & =\frac{1}{2}\langle\Psi, \Phi\rangle_{+}+\frac{1}{2}\langle\Psi, \Phi\rangle_{-} \\
& =\lim \sum_{i=0}^{n} \frac{\Psi\left(a_{i}\right)+\Psi\left(a_{l-1}\right)}{2}\left(\Phi\left(a_{i}\right)-\Phi\left(a_{l-1}\right)\right) .
\end{aligned}
$$

The limits are taken over finite sets $\left\{a_{0}, \ldots, a_{n}\right\}$ (with $a_{0}<a_{1}<\cdots<a_{n}$ ) ordered by inclusion. The limits for $\langle\Psi, \Phi\rangle_{ \pm}$exist by monotonicity if $\Phi, \Psi$ are real monotone and $\Phi$ is constant on $(\infty, a]$ and $[b, \infty)$. Therefore (using linear combinations and density) the limits exist in general.

Note that $\langle\Psi, \Phi\rangle$ depends only on the restriction of $\Psi$ to a small neighborhood of the support of $\Phi$. Also

$$
|\langle\Psi, \Phi\rangle| \leqq\|\Psi\|_{0} \operatorname{Var} \Phi
$$

Let $\mathscr{B}_{0}=\left\{\Phi \in \mathscr{B}: \lim _{|x| \rightarrow \infty} \Phi(x)=0\right\}$ and denote by $\Psi_{x}$ the characteristic function of $(-\infty, x)$. Using the linear form

we define

$$
\Psi \mapsto \alpha(\Psi)=\langle\Psi, \Phi\rangle,
$$

$$
\Phi_{\alpha}(x)=2 \alpha\left(\Psi_{x}\right)-\lim _{y \nearrow x} \alpha\left(\Psi_{y}\right)
$$

When $\Phi \in \mathscr{B}_{0}$, it is easily checked that $\Phi_{\alpha}=\Phi$. More generally if $\alpha: \mathscr{B} \rightarrow \mathbb{C}$ is linear and satisfies

$$
|\alpha(\Psi)| \leqq C_{\alpha}\|\Psi\|_{0},
$$

the function $x \mapsto \alpha\left(\Psi_{x}\right)$ has $\operatorname{Var} \leqq 2 C_{\alpha}$ and

$$
\operatorname{Var} \Phi_{\alpha} \leqq 6 C_{\alpha}
$$

[ $\Phi_{\alpha}$ is thus in $\mathscr{B}$, but not necessarily in $\mathscr{B}_{0}$. Furthermore it is not claimed that $\left\langle\Psi, \Phi_{\alpha}\right\rangle=\alpha(\Psi)$.]

B.2. Proof of part (a). Using the notation

we have

$$
\varphi_{\omega_{1} \cdots \omega_{m}}(x)=\varphi_{\omega_{1}}(x) \cdots \varphi_{\omega_{m}}\left(\psi_{\omega_{m-1}} \cdots \psi_{\omega_{1}} x\right)
$$

$$
\left\langle\Psi, \mathscr{M}^{m} \Phi\right\rangle=\sum_{\omega_{1} \cdots \omega_{m}}\left\langle\Psi, \varphi_{\omega_{1} \cdots \omega_{m}} \cdot\left(\Phi \circ \psi_{\omega_{m}} \circ \cdots \circ \psi_{\omega_{1}}\right)\right\rangle
$$


We may write

$$
\begin{aligned}
\left\langle\Psi, \varphi_{\omega_{1} \cdots \omega_{m}}\right. & \left.\left(\Phi \circ \psi_{\omega_{m}} \circ \cdots \circ \psi_{\omega_{1}}\right)\right\rangle \\
= & \sum_{k=1}^{m} \lim \sum_{i=1}^{m} \frac{1}{2}\left\{\left[\varepsilon_{\omega_{1}} \cdots \varepsilon_{\omega_{k-1}} \cdot\left(\varphi_{\omega_{1} \cdots \omega_{k-1}} \cdot \Psi\right) \circ \psi_{\omega_{1}}^{-1} \circ \cdots \circ \psi_{\omega_{k-1}}^{-1}\right]\left(a_{i}\right)\right. \\
& \cdot\left[\varphi_{\omega_{k+1} \cdots \omega_{m}} \cdot\left(\Phi \circ \psi_{\omega_{m}} \circ \cdots \circ \psi_{\omega_{k+1}}\right)\right]\left(\psi_{\omega_{k}} a_{l-1}+\operatorname{sym}\right\} \\
& \left.\cdot\left[\varphi_{\omega_{k}}\left(a_{l}\right)-\varphi_{\omega_{k}}\right)\left(a_{i-1}\right)\right] \\
+ & \lim \sum_{i=1}^{n} \frac{1}{2}\left\{\left[\varepsilon_{\omega_{1}} \cdots \varepsilon_{\omega_{m}} \cdot\left(\varphi_{\omega_{1} \cdots \omega_{m}} \cdot \Psi\right) \circ \psi_{\omega_{1}}^{-1} \circ \cdots \circ \psi_{\omega_{m}}^{-1}\right]\left(a_{i}\right)+\operatorname{sym}\right\} \\
& \cdot\left[\Phi\left(a_{i}\right)-\Phi\left(a_{l-1}\right)\right],
\end{aligned}
$$

where the "sym" terms are obtained by exchanging $a_{i}$ and $a_{i-1}$. Note that when the function $\psi_{\omega_{k-1}} \circ \cdots \circ \psi_{\omega_{1}}$ is decreasing, the change of variables that it defines interchanges "symmetric" terms and produces a negative sign (this is reflected in the factor $\varepsilon_{\omega_{1}} \cdots \varepsilon_{\omega_{k-1}}$ of the formula). We have thus

$$
\left|\left\langle\Psi, \mathscr{M}^{m} \Phi\right\rangle\right| \leqq \sum_{k=1}^{m}\left\|\widehat{\mathscr{M}}^{k-1} \Psi\right\|_{0}\left\|\mathscr{M}^{m-k} \Phi\right\|_{0} V+\left\|\widehat{\mathscr{M}^{m}} \Psi\right\|_{0} \operatorname{Var} \Phi
$$

Therefore if $\xi>\max (R, \widehat{R})$, there is $C>0$ such that

$$
\begin{aligned}
\left|\left\langle\Psi, \mathscr{M}^{m} \Phi\right\rangle\right| & \leqq C\left(m \xi^{m}\|\Psi\|_{0}\|\Phi\|_{0}+\xi^{m}\|\Psi\|_{0} \operatorname{Var} \Phi\right) \\
& \leqq(m+1) C \xi^{m}\|\Psi\|_{0} \operatorname{Var} \Phi
\end{aligned}
$$

hence

$$
\begin{aligned}
\operatorname{Var} \mathscr{M}^{m} \Phi & \leqq 6(m+1) C \xi^{m} \operatorname{Var} \Phi \\
\left\|\mathscr{M}^{m}\right\| & \leqq 6(m+1) C \xi^{m}
\end{aligned}
$$

and finally

$$
\text { spectral radius } \mathscr{M} \leqq \max (R, \widehat{R}) .
$$

B.3. Proof of part $(b)$. If $\left(K_{m}\right)$ is a sequence of operators of finite rank we have the general formula ${ }^{2}$

$$
\text { essential spectral radius of } \mathscr{M} \leqq \limsup _{m \rightarrow \infty}\left(\left\|\mathscr{M}^{m}-K_{m}\right\|\right)^{1 / m} \text {. }
$$

Let $\xi>\widehat{R}$; there is thus $C>0$ such that

$$
\left\|\widehat{\mathscr{M}}^{m}\right\|_{0} \leqq C \xi^{m}
$$

for all $m$. To prove (b) we will show that (for suitable $K_{m}$ ) we have

$$
\left\|\mathscr{M}^{m}-K_{m}\right\| \leqq P(m) \cdot \xi^{m},
$$

where $P(m)$ is a polynomial (of degree 1 ) in $m$.

${ }^{2}$ This is a relatively elementary fact, which constitutes the "easy" part of Nussbaum's essential spectral radius formula (Nussbaum [3]). 
We can choose a finite set $\Omega^{*} \subset \Omega$ so that the operator $\mathscr{M}^{*}$ defined by

$$
\left(\mathscr{M}^{*} \Phi\right)(x)=\sum_{\omega \in \Omega^{*}} \varphi_{\omega}(x) \Phi\left(\psi_{\omega} x\right)
$$

is arbitrarily close to $\mathscr{M}$. We have indeed

$$
\begin{gathered}
\left\|\mathscr{M}-\mathscr{M}^{*}\right\|,\left\|\widehat{\mathscr{M}}-\widehat{\mathscr{M}}^{*}\right\| \leqq \sum_{\omega \in \Omega \backslash \Omega^{*}} \operatorname{Var} \varphi_{\omega}, \\
\left\|\mathscr{M}^{*}\right\|,\left\|\widehat{\mathscr{M}}^{*}\right\|<V .
\end{gathered}
$$

We may thus take $\Omega^{*}$ (depending on $m$ ) such that

$$
\left\|\mathscr{M}^{k}-\mathscr{M}^{* k}\right\|,\left\|\widehat{\mathscr{M}}^{k}-\widehat{\mathscr{M}}^{* k}\right\| \leqq \xi^{k}
$$

for $k=1, \ldots, m$. The same estimates may be assumed to hold for the \|\|$_{0}$ operator norms; in particular we obtain

$$
\left\|\widehat{\mathscr{M}}^{* k}\right\|_{0} \leqq(C+1) \xi^{k}
$$

for $k=1, \ldots, m$.

For each $\omega \in \Omega^{*}$ we decompose $\Lambda_{\omega}$ into finitely many intervals $\Lambda_{(\omega, l)}$ and define a function $\bar{\varphi}_{\omega}$ with constant value $\varphi(\omega, i)$ in $\Lambda_{(\omega, i)}$. Taking $\varphi(\omega, i) \in \varphi_{\omega} \Lambda_{(\omega, i)}$ we have

$$
\operatorname{Var} \bar{\varphi}_{\omega} \leqq \operatorname{Var} \varphi_{\omega}
$$

Given $\delta>0$ we may also assume that the $\left.\Lambda_{(\omega, i}\right)$ are such that

$$
\left\|\varphi_{\omega}-\bar{\varphi}_{\omega}\right\|_{0}<\delta / \operatorname{card} \Omega^{*} \text {. }
$$

We define the operator $\overline{\mathscr{M}}$ by

$$
(\overline{\mathscr{M}} \Phi)(x)=\sum_{\omega \in \Omega^{*}} \bar{\varphi}_{\omega}(x) \Phi\left(\psi_{\omega} x\right),
$$

and obtain thus

$$
\begin{aligned}
\left\|\mathscr{M}^{*}-\overline{\mathscr{M}}\right\|_{0},\left\|\widehat{\mathscr{M}}^{*}-\widehat{\overline{\mathscr{M}}}\right\|_{0} \leqq \delta, \\
\|\overline{\mathscr{M}}\|,\|\overline{\hat{\mathscr{M}}}\|,\|\overline{\mathscr{M}}\|_{0}, \widehat{\overline{\mathscr{M}}} \leqq V .
\end{aligned}
$$

We may thus choose $\delta$ sufficiently small that

$$
\left\|\mathscr{M}^{* k}-\overline{\bar{M}}^{k}\right\|_{0},\left\|\widehat{\mathscr{M}}^{* k}-\widehat{\bar{M}}^{k}\right\|_{0} \leqq \xi^{k}
$$

for $k=1, \ldots, m$. In particular

$$
\left\|\widehat{\bar{M}}^{k}\right\|_{0} \leqq(C+2) \xi^{k}
$$

for $k=1, \ldots, m$.

We note that the linear form associated with $\mathscr{M}^{* m} \Phi$ is

$$
\begin{aligned}
\Psi \mapsto\left\langle\Psi, \mathscr{M}^{* m} \Phi\right\rangle= & \sum_{k=1}^{m} \lim \sum_{i=1}^{n} \sum_{\omega_{k}} \frac{1}{2}\left\{\left[(\widehat{\mathscr{M}} * k-1 \Psi)\left(a_{i}\right)\right] \cdot\left[\left(\mathscr{M}^{* m-k} \Phi\right)\left(\psi_{\omega_{k}} a_{i-1}\right)\right]\right. \\
& +\operatorname{sym}\} \cdot\left[\varphi_{\omega_{k}}\left(a_{i}\right)-\varphi_{\omega_{k}}\left(a_{i-1}\right)\right] \\
& +\lim \sum_{l=1}^{n} \frac{1}{2}\left\{\left(\widehat{\mathscr{M}}^{* m} \Psi\right)\left(a_{l}\right)+\operatorname{sym}\right\} \cdot\left[\Phi\left(a_{l}\right)-\Phi\left(a_{i-1}\right)\right] .
\end{aligned}
$$

This expression will be used in a moment. 
Let us denote by $\psi_{(\omega, i)}$ the restriction of $\psi_{\omega}$ to $\Lambda_{(\omega, i)}$. For fixed $k$ the intervals of definition of the $\psi_{\left(\omega_{m}, l_{m}\right)} \circ \cdots \circ \psi_{\left(\omega_{k+1}, i_{k+1}\right)}$ generate a partition of $\mathbb{R}$ into a finite set $\mathfrak{I}_{m-k}$ of intervals. Let $\mathfrak{I}_{m-k}^{\prime}$ be the set of interval endpoints, and $\mathfrak{I}_{m-k}^{\prime \prime}$, the set of interval interiors (this is a finite set of open intervals). For each $I \in \mathfrak{J}_{m-k}^{\prime \prime}$, choose $x_{I} \in I$ and define the operator $\mathscr{N}_{m-k}$ by

$$
\left(\mathscr{N}_{m-k} \Phi\right)(x)=\left\{\begin{array}{ll}
\left(\overline{\mathscr{M}}^{m-k} \Phi\right)(x) & \text { if } x \in \mathfrak{J}_{m-k}^{\prime} \\
\left\langle\Psi_{x_{I}}, \overline{\mathscr{M}}^{m-k} \Phi\right\rangle & \text { if } x \in I \in \mathfrak{J}_{m-k}^{\prime \prime}
\end{array} .\right.
$$

Finally we define the operator $K_{m}$ by

$$
K_{m} \Phi=\Phi_{\alpha},
$$

where $\Phi_{\alpha} \in \mathscr{B}$ is the function associated with the linear form $\alpha$ :

$$
\begin{aligned}
\Psi \mapsto \alpha(\Psi)= & \sum_{k=1}^{m} \lim \sum_{i=1}^{n} \sum_{\omega_{k}} \frac{1}{2}\left\{\left[\left(\widehat{\mathscr{M}}^{* k-1} \Psi\right)\left(a_{l}\right)\right] \cdot\left[\left(\mathscr{N}_{m-k} \Phi\right)\left(\psi_{\omega_{k}} a_{i-1}\right)\right]+\operatorname{sym}\right\} \\
& \cdot\left[\varphi_{\omega_{k}}\left(a_{i}\right)-\varphi_{\omega_{k}}\left(a_{l-1}\right)\right] .
\end{aligned}
$$

Therefore $K_{m}$ is of finite rank.

The values of $\overline{\mathscr{M}}^{m-k} \Phi-\mathscr{N}_{m-k} \Phi$ on the open interval $I \in \mathfrak{J}^{\prime \prime}{ }_{m-k}$ are determined by

$$
\overrightarrow{\mathscr{M}}^{m-k} \Phi(x)-\mathscr{N}_{m-k} \Phi(x)=2 \widetilde{\Phi}(x)-\lim _{y \nearrow x} \widetilde{\Phi}(y),
$$

where

$$
\widetilde{\Phi}(x)=\left\langle\Psi_{x}-\Psi_{x_{I}}, \overline{\mathscr{M}}^{m-k} \Phi\right\rangle=\left\langle\widehat{\overline{\mathscr{M}}}^{m-k}\left(\Psi_{x}-\Psi_{x_{I}}\right), \Phi\right\rangle,
$$

so that

$$
|\widetilde{\Phi}(x)| \leqq\left\|\widehat{\overline{\mathscr{M}}}^{m-k}\right\|_{0} \cdot \operatorname{Var} \Phi
$$

and

$$
\left\|\overline{\mathscr{M}}^{m-k} \Phi-\mathcal{N}_{m-k} \Phi\right\|_{0} \leqq 3\left\|\widehat{\overline{\mathscr{M}}}^{m-k}\right\|_{0} \operatorname{Var} \Phi \leqq 3(C+2) \xi^{m-k} \operatorname{Var} \Phi .
$$

Since we also have

$$
\left\|\mathscr{M}^{* m-k} \Phi-\overline{\mathscr{M}}^{m-k} \Phi\right\|_{0} \leqq \xi^{m-k}\|\Phi\|_{0}
$$

we find

$$
\left\|\mathscr{M}^{* m-k} \Phi-\mathcal{N}_{m-k} \Phi\right\|_{0} \leqq(3 C+7) \xi^{m-k} \operatorname{Var} \Phi
$$

By definition of $K_{m}$, we find that $\mathscr{M}^{* m} \Phi-K_{m} \Phi$ is the function associated with the linear form $\Psi \mapsto\left\langle\Psi, \mathscr{M}^{* m} \Phi\right\rangle-\alpha(\Psi)$. We have the estimate

$$
\begin{aligned}
\mid\langle\Psi, & \left.\mathscr{M}^{* m} \Phi\right\rangle-\alpha(\Psi)\left|\leqq \sum_{k=1}\right| \lim \sum_{l=1}^{n} \sum_{\omega_{k}} \frac{1}{2}\left\{\left[\left(\widehat{\mathscr{M}}^{* k-1} \Psi\right)\left(a_{l}\right)\right]\right. \\
& \left.\times\left[\left(\mathscr{M}^{* m-k} \Phi-\mathscr{N}_{m-k} \Phi\right)\left(\psi_{\omega_{k}} a_{i-1}\right)\right]+\operatorname{sym}\right\} \cdot\left[\varphi_{\omega_{k}}\left(a_{i}\right)-\varphi_{\omega_{k}}\left(a_{i-1}\right)\right] \mid \\
& +\left|\lim \sum_{i=1}^{n} \frac{1}{2}\left\{\left(\widehat{\mathscr{M}}^{* m} \Psi\right)\left(a_{l}\right)+\operatorname{sym}\right\} \cdot\left[\Phi\left(a_{l}\right)-\Phi\left(a_{l-1}\right)\right]\right| \\
& \leqq \sum_{k=1}^{m}(C+1) \xi^{k-1}\|\Psi\|_{0} \cdot(3 C+7) \xi^{m-k} \operatorname{Var} \Phi \cdot V+(C+1) \xi^{m}\|\Psi\|_{0} \cdot \operatorname{Var} \Phi \\
& =\left(m C^{\prime}+C+1\right) \xi^{m}\|\Psi\|_{0} \operatorname{Var} \Phi
\end{aligned}
$$


and

$$
\left\|\mathscr{M}^{m}-K_{m}\right\| \leqq \xi^{m}+6\left(m C^{\prime}+C+1\right) \xi^{m}=P(m) \cdot \xi^{m}
$$

with $P(m)=6 m C^{\prime}+6 C+7$, of degree 1 in $m$ as announced.

B.4. Proof of part (c). We refer to [4] where a similar result is proved. The proof given in [4] also applies here, with inessential modifications.

\section{References}

1. Baladi, V., Ruelle, D.: An extension of the theorem of Milnor and Thurston on the zeta functions of interval maps. Ergod. Th. and Dynam. Syst. 14, 621-632 (1994)

2. Milnor, J., Thurston, W.: On iterated maps of the interval. In: Dynamical systems, Alexander, J.C. (ed.). Lecture Notes in Mathematics N 1342. Berlin: Springer, 1988, pp. 465-563

3. Nussbaum, R.D.: The radius of the essential spectrum. Duke Math. J. 37, 473-478 (1970)

4. Ruelle, D.: Spectral properties of a class of operators associated with maps in one dimension. Ergod. Th. and Dynam. Syst. 11, 757-767 (1991)

Communicated by J.-P. Eckmann 